\title{
The Impossible Process: Thermodynamic Reversibility
}

\author{
John D Norton ${ }^{1}$ \\ Department of History and Philosophy of Science \\ Center for Philosophy of Science \\ University of Pittsburgh \\ jdnorton@pitt.edu
}

\begin{abstract}
Standard descriptions of thermodynamically reversible processes attribute contradictory properties to them: they are in equilibrium yet still change their state. Or they are comprised of non-equilibrium states that are so close to equilibrium that the difference does not matter. One cannot have states that both change and no not change at the same time. In place of this internally contradictory characterization, the term "thermodynamically reversible process" is here construed as a label for a set of real processes of change involving only non-equilibrium states. The properties usually attributed to a thermodynamically reversible process are recovered as the limiting properties of this set. No single process, that is, no system undergoing change, equilibrium or otherwise, carries those limiting properties. The paper concludes with an historical survey of characterizations of thermodynamically reversible processes and a critical analysis of their shortcomings.
\end{abstract}

${ }^{1}$ My special thanks to Giovanni Valente. His critical analysis of Norton (2014), both in discussion and in an unpublished manuscript, occasioned this paper. I also thank Wayne Myrvold, Jos Uffink and an anonymous referee for extensive, productive discussion. Philip Ehrlich helped with non-standard analysis. 


\section{Introduction}

In studies of the conceptual foundations of thermodynamics, the perpetually troublesome notion of entropy attracts almost all the attention. There is a second notion that is just as essential to thermodynamics and just as troublesome, yet it is largely overlooked. This is the notion of thermodynamically reversible or quasi-static processes. They are used in the definition of entropy and are distinguished as the least dissipative of processes. They are, loosely speaking, processes whose driving forces-temperature differences, pressure differences and the like-are balanced so delicately that they could proceed equally easily in either direction, reversing the quantities of heat and work exchanged. The core difficulty is immediately visible. The processes are supposed to conform to two contradictory requirements, which are encapsulated in a paradox.

\section{Paradox of Thermodynamically Reversible Processes}

1. They are processes with a non-equilibrium imbalance of driving forces, such as non-zero temperature differences or unbalanced mechanical forces; for this imbalance is needed to move the system from one state to another.

2. At the same time they are sets of equilibrium states in which, by definition, there is no imbalance of forces; for then the forward and the reverse processes pass through the same set of equilibrium states and both can be represented by the same curve in equilibrium state space.

A system cannot both be out of equilibrium and in equilibrium at the same time. The difficulty has been recognized since the beginning of thermodynamics. Yet virtually all efforts to deal with it involve inadequate deflections that merely give the appearance of a solution. An "infinitely slow" process is supposed to be one that changes, while its states always remain in equilibrium. Yet a process that is infinitely slowed in all its stages is one that never progresses past any stage. An "insensible" or "infinitesimally small" disequilibrium is supposed to bring us a non-zero driving force, so that the state of the system is out of equilibrium and changes in time, while also remaining in equilibrium. The minuscule departure from equilibrium is supposed too small to matter. Yet, no matter how small, it does matter, since this departure is essential to secure a process that changes in time.

It is to no avail. Incantations of "infinitely slow," "insensible" and "infinitesimal" have no magical powers that overturn the law of the excluded middle. Either a system is in 
equilibrium or it is not; it cannot both be both. Either a system undergoes change or it does not; it cannot do both. ${ }^{2}$

Almost all developments of thermodynamics address the problem. But, as we shall see at some length, they do so in haste, on the way to more important matters. The secondary literature has rarely objected. Rechel (1947) is an exception that identifies forcefully just the sort of contradictions to be discussed here. More recently, Uffink (2001) provides a sustained critique that reveals many of the tensions in the literature. As an illustration of a more general account of idealizations and approximations, Norton (2014) has sought to resolve the problem by denying that thermodynamically reversible processes are idealized processes at all. Hence there is no process, fictional or otherwise, that is posited to bear contradictory properties. Rather all we have are approximations, that is, descriptions of the limiting behavior of a set of many, real, nonequilibrium processes.

\section{What is to Come}

Part I of this paper, following Norton (2014), develops a positive account of thermodynamically reversible processes that is designed to resolve the above paradox. It is developed in Section 2. Its founding idea is that there is no single process that can be identified as a thermodynamically reversible process. A set of equilibrium states is no process at all, since the states do not change in time. A process constituted of near equilibrium states is more promising, since these states do change in time. The difficulty is to know just how close to equilibrium its states must come.

This last difficulty is resolved in the proposal by representing a reversible process not by a single process "close enough" to equilibrium, but by continuous sets of irreversible processes, whose non-equilibrium states come arbitrarily close to equilibrium states, while never actually becoming equilibrium states. This limiting set of equilibrium states is just a curve in equilibrium

\footnotetext{
2 Alternatively, one can simply dispense with one disjunct and consider what I call below the "bare" form of "quasi-static processes." They are by definition merely sets of unchanging equilibrium states. The problem returns, however, when one has to connect results about this set with dynamical processes of change in non-equilibrium thermal systems.
} 
state space. It forms the unrealized boundary of the non-equilibrium states in the set of processes that do evolve in time, in both forward and reversed directions.

The properties normally associated with a reversible process are recovered from the set of irreversible processes through limit operations. These limits return vanishing driving forces and the requisite quantities of heat and work. Crucially, the limit operations generate limit properties only. They do not generate a single process that carries these properties, for these properties are mutually incompatible: if the driving forces vanish, there can be no heat transferred or work done. By this means, the account avoids the paradoxical fiction that the thermodynamics literature has tried so hard to realize: a single process, evolving in time yet comprised of unchanging equilibrium states.

Section 3 will connect this last analysis with general ideas concerning idealizations and approximations. Drawing on an account given elsewhere of idealizations produced by limits, I argue that, in this case, there is no well-behaved limit process produced when we let thermodynamic driving forces go to zero. Rather we should conceive the notion of a reversible process as an approximation, according to a specific use of the term "approximation."

Section 4 will seek to demonstrate that this new characterization of thermodynamically reversible processes is adequate for thermodynamic theorizing. In the existing literature, it is standard to derive results by means of the fiction of a reversible process of equilibrium states that are supposed to evolve in time, even though equilibrium states are unchanging. The new derivations will use only irreversible processes constituted of non-equilibrium states, drawn from the set specified in the definition of Section 2.

Whether there exist sets of irreversible processes conforming with the definition of Section 2, depends upon the background dynamical theory in which the processes occur. They do not exist in one important case, recounted in Section 5. For molecular systems on molecular scales, thermal fluctuations preclude the existence of irreversible process that can be completed and, at the same time, have states that can be brought arbitrarily close to equilibrium states.

Part II of this paper seeks to rectify an imbalance in the present literature. Considerable efforts have been spent on understanding the sometimes elusive notion of entropy. The notion of a thermodynamically reversible process is just as important to thermodynamics and the second law. Thermodynamics flourished for decades without the notion of entropy. It did so, first in the work on Carnot of 1824, and then in the founding of the modern theory in the work of Clausius 
and Thomson in the early 1850s. It was only a decade later that the notion of entropy was introduced by Clausius in 1865 . Yet, compared to entropy, the notion of thermodynamically reversible processes receives scant attention in the critical and historical literature.

Section 6 of Part II will sketch a striking analogy to the notion of a reversible process in the work of Sadi Carnot's father, Lazare Carnot, on the efficiency of ordinary machines. Section 7 will them provide a survey of characterizations of thermodynamically reversible processes in the literature. One goal of the survey is to document the range of proposals. A second goal is critical. I will argue that none of proposals is entirely adequate. All of them, ${ }^{3}$ even the most cautious, are subject to one or other form of the above paradox. My conclusion is not that that the problems are irresolvable. I offer the analysis of Section 2 as a serviceable resolution. Rather it is that there is a near universal practice in the present literature of defining thermodynamically reversible processes in haste, so that something like what is proposed seems credible, while what is actually said is not. The concluding Section 8 reviews briefly how the proposal of Section 2 escapes the problems troubling characterizations of reversible processes presently in the literature.

\section{Part I. What Thermodynamically Reversible Processes Really Are}

\section{Thermodynamically Reversible Processes as Sets of Irreversible Processes}

\subsection{Properties Required}

The following are the properties normally associated with a thermodynamically reversible process. They will form the basis of a characterization free of manifest paradox:

They are processes that can proceed in either the forward or reverse direction, since their thermodynamic forces are in near perfect balance.

\footnotetext{
3 I exclude an account by Duhem in Section 2.3.
} 
If heat $\mathrm{Q}_{\mathrm{f}}$ is gained by the system and work $\mathrm{W}_{\mathrm{f}}$ is done by the system in the forward process, then heat $\mathrm{Q}_{\mathrm{r}}=-\mathrm{Q}_{\mathrm{f}}$ is gained by the system and work $\mathrm{W}_{\mathrm{r}}=-\mathrm{W}_{\mathrm{f}}$ is done by the system in the reverse process.

If a space of equilibrium states is available, then the process can be represented by a continuous curve in the space.

The rate at which work is done is given by

$$
\mathrm{dW}=\sum_{\mathrm{i}} \mathrm{X}_{\mathrm{i}} \mathrm{dx}
$$

This expression (1) is derived from a standard statement of the first law of thermodynamics

$$
\mathrm{dU}=\mathrm{dQ}-\mathrm{dW}=\mathrm{dQ}-\sum_{\mathrm{i}} \mathrm{X}_{\mathrm{i}} \mathrm{d} \mathrm{x}_{\mathrm{i}}
$$

$\mathrm{U}$ is the system internal energy and the paired variables $\mathrm{X}_{1}$ and $\mathrm{x}_{1}, \mathrm{X}_{2}$ and $\mathrm{x}_{2}$, etc. are pairs of generalized force $\left(\mathrm{X}_{\mathrm{i}}\right)$ and displacement $\left(\mathrm{x}_{\mathrm{i}}\right)$ variables. Common pairings are pressure $\mathrm{P}$ and volume $\mathrm{V}$, surface tension $\gamma$ and surface area $\mathrm{A}$, as well as magnetic and electric quantities such as magnetic field and magnetic moment; and electric field strength and dipole moment. (See Pippard, 1966. pp. 23-28.) The operator $d$ is usually represented as marking a small or even infinitesimal difference in the quantity on which it acts. A mathematically cleaner reading is to assign a path parameter $\lambda$ to the process and identify the operator as $d=d / d \lambda$.

Here the term "driving force" denotes temperature differences and these generalized forces. They are well-defined in non-equilibrium systems. Fourier's equation for the dynamics of heat transport employs temperature gradients as a force that drives heat flow. The Navier Stokes equation employs pressure gradients as a force that drives momentum in fluids. More generally, external to thermodynamics, pressure and surface tension are well-defined in theories of the mechanics of fluids; and electric and magnetic field are well-defined in electrodynamics.

\subsection{The Characterization}

These properties are all captured by the following:

\section{Definition}

The label "thermodynamically reversible process" denotes a set of irreversible processes in a thermal system, delimited by the set of equilibrium states in (d) such that: 
(a) Each process may exchange heat or work with its surroundings, because of imbalanced driving forces (temperature differences, generalized forces).

(b) The processes can be divided into a "forward" and a "reverse" set such that the total heat gained and the total work done have opposite signs in the two sets.

(c) In each set, there are processes in which the net driving forces are arbitrarily small. In the case of generalized forces, the net driving force is the difference between the generalized force and the force in the surrounding system that counteracts it. ${ }^{4}$

(d) Under the limit ${ }^{5}$ of these net driving forces going to zero, the states of both forward and reverse processes approach the same set of equilibrium states and these states form a curve in equilibrium state space.

(e) The limiting values of heat gained and work done by the forward process are $Q_{f}$ and $\mathrm{W}_{\mathrm{f}}$; and by the reverse process $\mathrm{Q}_{\mathrm{r}}$ and $\mathrm{W}_{\mathrm{r}}$; and they satisfy

$$
\mathrm{Q}_{\mathrm{f}}=-\mathrm{Q}_{\mathrm{r}} \text { and } \mathrm{W}_{\mathrm{f}}=-\mathrm{W}_{\mathrm{r}}
$$

(f) These limiting quantities of heat and work, computed at any stage of the process, correspond to those computed by integration of the relations (1) and (2) along the curve of the equilibrium states in equilibrium state space.

While they may come arbitrarily close, none of the states of the irreversible processes are exactly equilibrium states. For otherwise the processes cannot complete in any finite time. The set is represented in Figure 1.

4 For example, the generalized driving force in an expanding gas is its pressure and the counteracting force is the weight against which the gas pressure acts. The net driving force for heat transfer is just the temperature difference.

5 To define the sequence of processes of successively smaller driving forces used in the limit, we say that process $\mathrm{A}$ has smaller driving forces than process $\mathrm{B}$ if the greatest temperature difference and generalized thermodynamic force in A is less than the corresponding maxima of B. The driving forces go to zero if these maxima go to zero. 


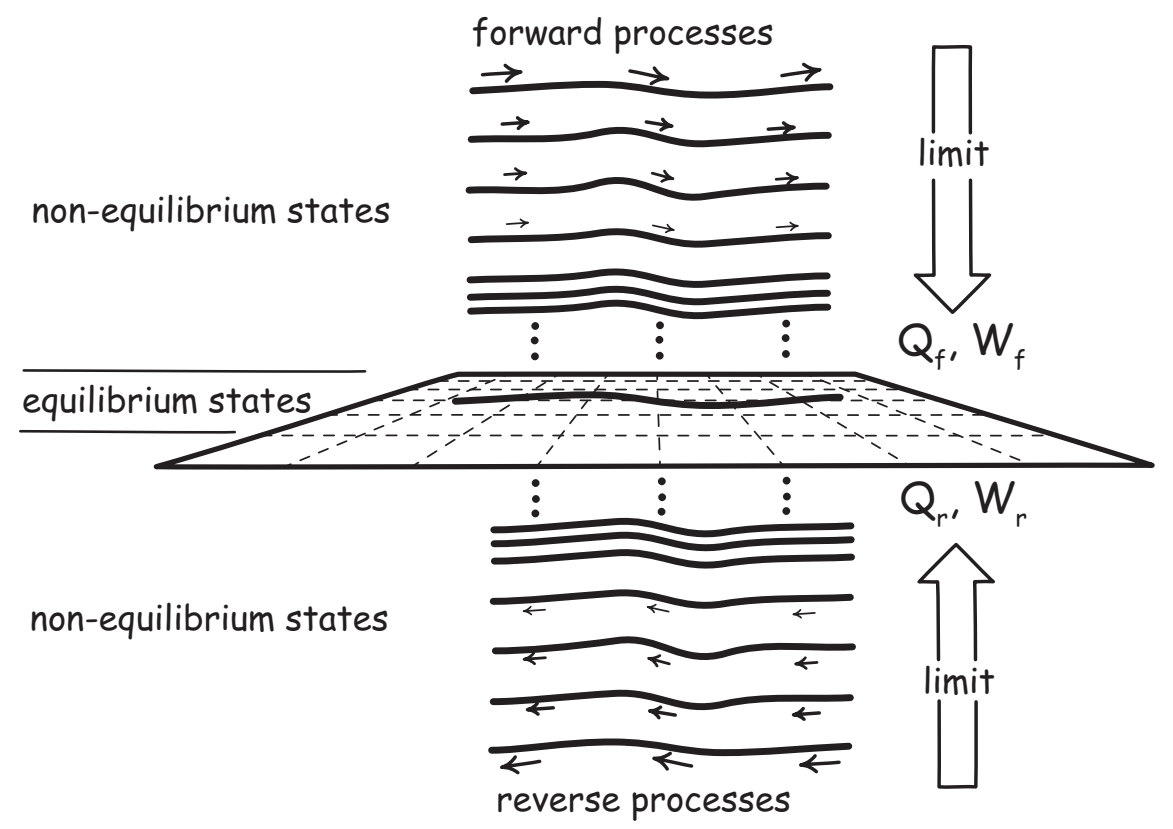

Figure 1. Set of Processes Forming a Reversible Process

The definition requires an additional assumption if it is to be used in thermodynamics:

\section{Existence 6}

There is a thermodynamically reversible process for any curve in equilibrium state space.

Whether the existence assumption is true depends upon the particular dynamical theory that governs changes in the thermal systems. It can fail. We shall see below in Section 5 that real processes at molecular scales cannot be brought arbitrarily close to sequences of equilibrium states. At these scales, there are no thermodynamically reversible processes.

Finally, this analysis can be applied to heat engines, since they consist of a sequence of thermal processes. A reversible heat engine is constructed from the corresponding sequence of thermodynamically reversible processes.

\footnotetext{
6 This existence postulate does not contradict Carathéodory's inaccessibility postulate since the latter is restricted to adiabatic processes.
} 


\subsection{Duhem's Analysis}

This last analysis seems both natural and obvious, so it was puzzling that it is not present in the literature. After a first draft of this paper was completed, it was with some relief that I found essentially this account had been developed quite carefully by Duhem (1903, pp. 59-74). He emphasized that the equilibrium states associated with a reversible process are merely an unrealized common boundary of the states passed by the real, non-equilibrium, forward and reverse processes. Duhem first gives his characterization in an analysis of Atwood's machine, in which a weight is slowly raised and lowered by a counterweight (p. 70, emphasis in original):

This series of equilibrium states $\alpha, \beta, \gamma, \delta, \ldots$ which is passed over by no modification of the system is, in some sort ${ }^{[7]}$, the common boundary of the real transformations that bring the system from the state 1 to the state 2 and of the real transformations that bring the system from state 2 to state $1 ; \ldots$ this series of equilibrium states is called a reversible transformation.

Thus the reversible transformation is a continuous series of equilibrium states; it is essentially unrealizable; but we may give our attention to these equilibrium states successively either in the order from state 1 to state 2, or in the reverse order; this purely intellectual operation is denoted by these words: to cause a system to undergo the reversible transformation considered, either in the direction 1-2, or in the reverse direction.

Duhem then illustrates carefully his central claim that the reversible process consists merely of common boundary states of real processes in three examples: vaporization of a liquid, dissociation of cupric oxide and dissociation of water vapor. Trevor (1927, pp. 16-19) appears to give essentially the same account and even uses the (unnamed) Atwood machine to illustrate it. That suggests he drew the account from Duhem, although Duhem is not cited. Trevor had earlier translated Duhem (1898) into English.

Duhem's (1903, pp. 59-74) account is a simplified and expanded development of his earlier writings on reversible processes. Needham (2011, pp. vi-vii) provides a brief overview of

7 JDN: "en quelque sorte" is better translated here as "as it were." 
this work in his editor's introduction to a volume that translates many of Duhem's papers. See also Needham (2013, pp. 406-407).

\section{Idealizations created by Limits}

That such an account of reversible processes is possible is foreshadowed by common remarks in the literature. It is accepted that thermodynamically reversible processes involve physical impossibilities. The awkwardness is then excused by calling them idealizations. Goodenough (1911, p. 49) writes

Strictly speaking, there are no reversible changes in nature. We must consider reversibility as an ideal limiting condition that may be approached but not actually attained when the processes are conducted very slowly.

Zemansky (1968,p. 196) is more explicit:

Since it is impossible to satisfy these two conditions [of reversibility] perfectly, it is obvious that a reversible process is purely an ideal abstraction, extremely useful for theoretical calculations (as we shall see) but quite devoid of reality. In this sense, the assumption of a reversible process in thermodynamics resembles the assumptions made so often in mechanics, such as those which refer to weightless strings, frictionless pulleys, and point masses.

The idea is initially appealing. As Zemansky points out, it is quite standard to introduce idealizations in physics by taking limits. We take the limit of ever lighter strings until they are weightless; of pulleys with diminishing friction until they are frictionless; and of masses of diminishing size until they are mere points. We proceed to theorize with these structures even though we know there are no weightless strings, frictionless pulleys and point masses.

Commonly these limiting procedures do not produce foundational problems. But merely declaring something an idealization produced by taking a limit is no guarantee that the result is well-behaved. The result need not be. A point charge, even in ordinary electrostatics, carries an infinite field energy that may, in some circumstances, call for renormalization.

Thermodynamically reversible processes turn out to be just such a troublesome case in which the uncritical taking of limits brings disaster. To see why, we need to look more closely at how taking limits, if done unreflectively, can lead to trouble. 


\subsection{A Limit System May Not Carry the Limit Property}

When we pursue a sequence of systems to a limit, we have the following structure. First there is a sequence of systems that approaches some limit system:

$$
\text { system }_{1}, \text { system }_{2}, \text { system }_{3}, \ldots \rightarrow \text { limit system }
$$

Then there is a corresponding sequence of properties of the systems that approached some limit property:

$$
\text { property }_{1} \text {, } \text { property }_{2} \text {, } \text { property }_{3}, \ldots \rightarrow \text { limit property }
$$

Generally the two match up well: the limit system carries the limit property. When this matching fails, different sorts of pathologies arise. Sometimes there is no limit system at all. Such is the case as we take the limit of ever larger spheres in ordinary space. Loosely speaking, the limit is an infinite sphere. That is nonsense, of course. There is no "infinite sphere" in ordinary Euclidean space.

The anomaly that is most prone to mislead arises when the limit property fails to match the properties of the limit system and we overlook the mismatch. Norton (2012) has cataloged examples. The most important pertains to the various "thermodynamic limits" employed in statistical physics. In them, one considers systems of components, such as molecules, of ever greater number. As the component number grows without limit, the thermal properties of the systems stabilize to well-behaved limiting values. It is tempting to presume that these same properties are carried by the infinite limit system that consists of infinitely components. However that is generally not so. Infinite systems of molecules are generically indeterministic and their behavior is, as a result, not assured to resemble that of thermal systems with very many, but still finitely many components.

Precisely this pathology arises in the case of thermodynamically reversible processes. We have a sequence of irreversible processes, each of which is slowed by diminishing the driving forces. Each process carries the property of completing a change, while requiring ever more time to do it. The limit of this property is the property of completing a change. The limit approached by the processes themselves, however, is no process at all. It is merely a static set of states in equilibrium that no longer carry the limit property of completing the change.

There is a mismatch of the limit of the properties of the irreversible processes and the properties of limit of the irreversible process. The repeated error of the literature described in 
Part II below is to try to argue away that mismatch and to pretend that the property of completing the change can still somehow be attributed to the limit of the processes that consists of an unchanging set of equilibrium states.

\subsection{Limits are not Properties of the Limit System but of a Set of Systems}

If the key property of completing a change does not belong to the limit approached of unchanging equilibrium states, to what does it belong? The property of completion is borne by the set of irreversible processes used in taking the limit, but not by the limit system itself, which is just a set of unchanging equilibrium states.

This last fact directs how thermodynamically reversible processes are to be understood. The term does not refer to a single process. The attempts to make it refer to one lead to the paradox above. Rather, the term refers to a set of irreversible processes; and the properties normally attributed to thermodynamically reversible processes are really borne as limiting properties of this set.

\subsection{Approximations, not Idealizations}

In Norton $(2014, \S 3)$ I described this situation by denying that thermodynamically reversible processes are idealizations, but asserting that they are really approximations.

That denial depends on the characterization given in Norton (2012) of idealizations. They are independently definable but possibly fictitious systems; and an exact description of their properties is also an inexact description of the real processes of interest. Since no process, real or fictitious, can carry all the properties of thermodynamically reversible processes without paradox, these processes cannot be idealizations. They are not consistently definable.

Rather, talk of thermodynamically reversible processes provides what are called in Norton (2012) approximations: inexact propositional descriptions of the systems of interest. Those systems of interest are real, irreversible processes with very small driving forces; and they have the property, for example, of passing heat very slowly with a very small temperature difference. The limit of this property is the transferring of heat with no temperature difference at all. A description of this limit property, the unrealizable

$$
\text { "...transferring heat ... with no temperature difference..." }
$$

is an inexact description of a real system that is correctly described as 
“...transferring heat ...with very small temperature difference...”.

The properties recovered from this limiting operation serve only to provide such inexact descriptions; and nothing more. They cannot consistently describe any process exactly, for no process can conform with them exactly. No process can transfer heat when there is no temperature difference.

\section{What Thermodynamically Reversible Processes Must Do}

We can see that the characterization of thermodynamically processes of Section 2 is adequate by showing that its supports rederivation of the results traditionally derived using the notion of thermodynamically reversible processes. Sommerfeld's (1956, p. 19) remark foreshadows the character of the rederivations when he wrote:

In spite of their not being real, reversible processes are most important in thermodynamics because definite equations can be obtained only by considering reversible changes; irreversible changes can only be described with the aid of inequalities when equilibrium thermodynamics is used.

What Sommerfeld does not mention is that, if one has enough inequalities, they can form equalities, as we shall now see. The derivations are essentially the familiar ones, but complicated slightly by the use of inequalities.

\subsection{The Efficiency of Heat Engines}

The extraordinary innovation of Carnot's (1824) essay was a means of inferring quite general limits on the efficiency of heat engines. He pictured heat engines as deriving mechanical effect by passing heat from a hot place to cold place. He was able to show that the greatest mechanical effect for any two such places was generated by a heat engine that could be operated in reverse and that all such reversible engines performed equally well, independently of their construction and whether their working material was steam, air or anything else. Carnot's original analysis was conducted within the caloric theory of heat under the supposition that heat is conserved during its passage from hot to cold. Subsequent analysis by Clausius (1851) and Thomson (1852) modified Carnot's analysis by requiring that the work produced by a heat 
engine resulted from the conversion of some of the heat into work. These papers mark the birth of the modern theory of thermodynamics.

Carnot's result is stated in terms of the internally contradictory notion of a reversible heat engine that could transfer heat without a temperature difference. We will change that. A heat engine is a sequence of thermodynamically reversible processes that draws and discharges heat from and to a hot place and cold place, while converting some of the heat energy into work. Combining them, this engine is just a single, lengthy thermodynamically reversible process. Below we will treat this reversible engine as the corresponding set of irreversible process, as specified in Section 2 above.

The efficiency $\eta$ of a heat engine is defined in the usual way $\eta=W / Q$, where $W$ is the work delivered by the engine and $Q$ the heat drawn from the hot place. A reversible heat engine consists of a set of irreversible processes that form a reversible process as defined in Section 2.2. Its efficiency $\eta_{\text {rev }}$ is defined as the ratio of the limiting values $W_{f}$ and $Q_{f}$ for $W$ and $Q$ as given in (e) above. There is no assumption that any individual engine or processes in the set can realize this efficiency.

\subsection{Reversible Engines are the Most Efficient}

First we show that no irreversible heat engine working between the same temperatures can have a higher efficiency than the efficiency of a reversible engine. To show this, assume for purposes of reductio that we have an irreversible engine whose efficiency $\eta_{i r r}=W_{i r r} / Q_{i r r}$ is greater than that of a reversible engine $\eta_{\mathrm{rev}}=\mathrm{W}_{\mathrm{f}} / \mathrm{Q}_{\mathrm{f}}=\mathrm{W}_{\mathrm{r}} / \mathrm{Q}_{\mathrm{r}}$. That is

$$
\eta_{\text {irr }}>\eta_{\text {rev }}
$$

As noted, we do not assume that any real heat engine can operate with the efficiency $\eta_{\text {rev }}$. However the definition of a reversible process allows that an irreversible heat engine can come arbitrarily close in its operation. Moreover, since the process is reversible, these irreversible heat engines are found in both the "forward" and "reverse" sets of irreversible processes. That is, there is a sequences of processes in the reverse set that delivers heat $\mathrm{Q}$ to the hot place and consumes work $\mathrm{W}$, such that

$$
\mathrm{W} / \mathrm{Q}=\eta_{\mathrm{rev}}-\varepsilon
$$


where the non-zero $\varepsilon$ can be brought arbitrarily close to zero by suitable selection of processes in the set. We can scale the operations of the two engines so that $\mathrm{W}_{\mathrm{irr}}=\mathrm{W}$ and couple the two engines in the usual way: work from the irreversible engine is used to operate the reversed engine, as shown in Figure 2.
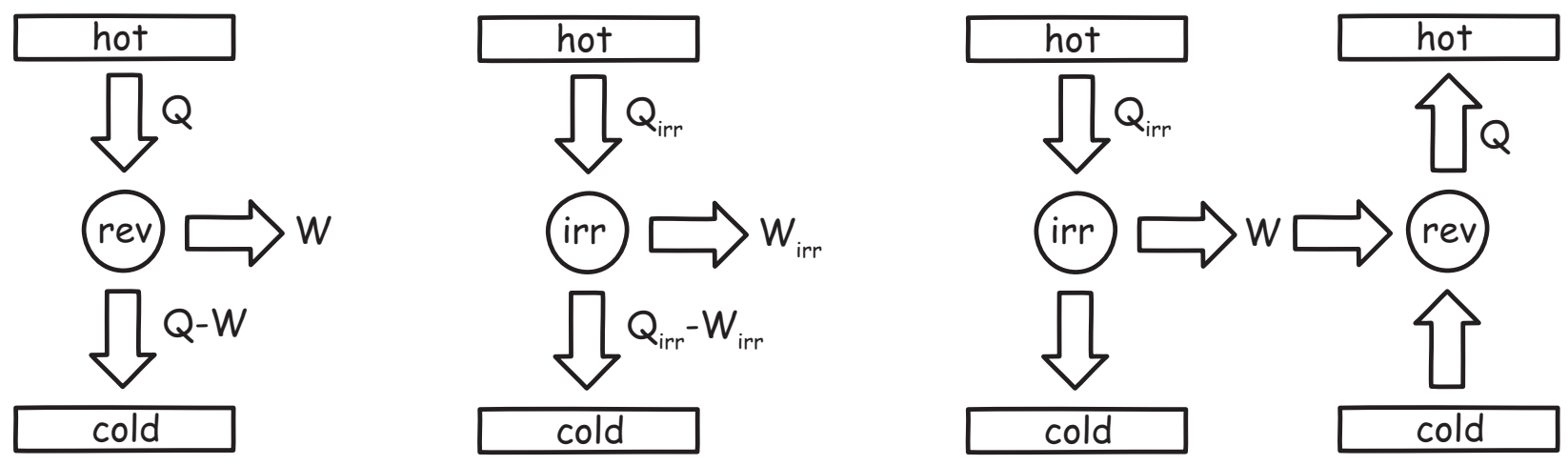

Figure 2. Coupled Reversible and Irreversible Heat Engines.

That is, we have $\mathrm{W}=\mathrm{Q}\left(\eta_{\mathrm{rev}}-\varepsilon\right)=\mathrm{Q}_{\mathrm{irr}} \eta_{\text {irr }}=\mathrm{W}_{\text {irr }}$, so that

$$
\frac{Q}{Q_{i r r}}=\frac{\eta_{i r r}}{\eta_{r e v}-\varepsilon}>1
$$

The second inequality arises since we can make $\varepsilon$ arbitrarily small and we have assumed that $\eta_{\text {irr }}>\eta_{\text {rev }}$. The net effect of the combined operation of the two engines is merely to take heat $Q$ $\mathrm{Q}_{\text {irr }}$ from the cold place and deliver it to the hot place. If $\mathrm{Q} / \mathrm{Q}_{\mathrm{irr}}>1$, then this quantity of heat $\mathrm{Q}$ $\mathrm{Q}_{\text {irr }}$ is a positive quantity. This is a violation of the Clausius form of the second law of thermodynamics, for we have a process whose sole effect is to move heat from cold to hot without the net consumption of work.

This contradiction completes the reductio. No irreversible heat engine can be more efficient than the efficiency assigned to a reversible engine.

\subsection{All Reversible Engines Have the Same Efficiency}

Similar inferences allow us to show that all reversible heat engines operating between the same temperatures have the same efficiency. As before, a reversible heat engine is a set of processes as defined in Section 2.2. There is no assumption that any process realizes the reversible efficiency $\eta_{\text {rev }}$. 
Assume for purposes of reductio that we have two such reversible engines, A and B, that operate at different efficiencies, with the first more efficient than the second. That is,

$$
\eta_{\mathrm{A}}=\mathrm{W}_{\mathrm{A}, \mathrm{f}} / \mathrm{Q}_{\mathrm{A}, \mathrm{f}}>\mathrm{W}_{\mathrm{B}, \mathrm{f}} / \mathrm{Q}_{\mathrm{B}, \mathrm{f}}=\eta_{\mathrm{B}}
$$

Hence we can find real engines that operate at efficiencies within arbitrarily small, non-zero $\varepsilon_{\mathrm{A}}$ and $\varepsilon_{\mathrm{B}}$ of these unrealizable efficiencies, with quantities of work and heat:

$$
\mathrm{W}_{\mathrm{A}} / \mathrm{Q}_{\mathrm{A}}=\eta_{\mathrm{A}}-\varepsilon_{\mathrm{A}} \quad \mathrm{W}_{\mathrm{B}} / \mathrm{Q}_{\mathrm{B}}=\eta_{\mathrm{B}}-\varepsilon_{\mathrm{B}}
$$

We scale the operations of the two engines so that $\mathrm{W}=\mathrm{W}_{\mathrm{A}}=\mathrm{W}_{\mathrm{B}}$. They can now be coupled so that work from engine $\mathrm{A}$ is used to drive the second less efficient engine $\mathrm{B}$ in reverse, as shown in Figure 3. Engine $A$ draws heat $Q_{A}$ from the hot place and engine $B$ returns heat $Q_{B}$ to it. There is a net transport of heat $\mathrm{Q}_{\mathrm{B}}-\mathrm{Q}_{\mathrm{A}}$ from the cold place to the hot place, with no net work exchanged with the surroundings.
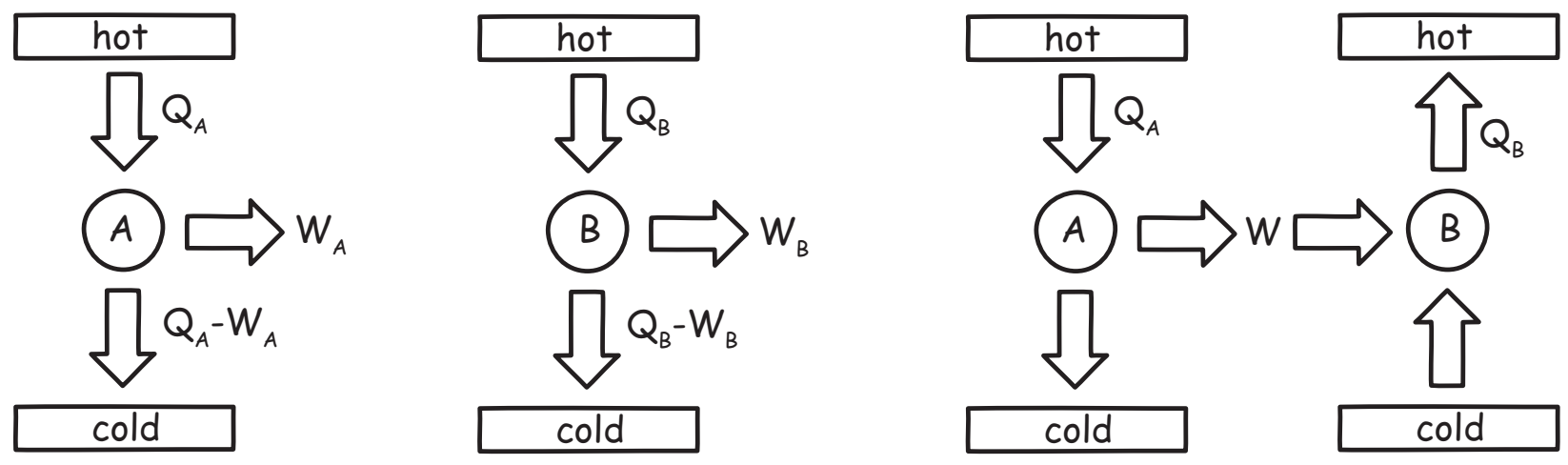

Figure 3. Coupled Reversible Heat Engines

The equalities $\mathrm{W}=\mathrm{W}_{\mathrm{A}}=\mathrm{W}_{\mathrm{B}}$ allows us to recover

$$
\mathrm{W}=\mathrm{W}_{\mathrm{A}}=\left(\eta_{\mathrm{A}}-\varepsilon_{\mathrm{A}}\right) \mathrm{Q}_{\mathrm{A}}=\left(\eta_{\mathrm{B}}-\varepsilon_{\mathrm{B}}\right) \mathrm{Q}_{\mathrm{B}}=\mathrm{W}_{\mathrm{B}}
$$

so that

$$
\frac{Q_{B}}{Q_{A}}=\frac{\left(\eta_{A}-\varepsilon_{A}\right)}{\left(\eta_{B}-\varepsilon_{B}\right)}>1
$$

The second inequality arises since we can make $\varepsilon_{A}$ and $\varepsilon_{B}$ as small as we like, while $\eta_{A}$ and $\eta_{B}$ are fixed in value with $\eta_{A}>\eta_{B}$. It follows that $Q_{B}-Q_{A}>0$. The net effect of the cycle is to transfer heat from hot to cold without any net consumption of work. The Clausius form of the second law of thermodynamics is violated and the reductio is complete. 
Analogous arguments gives the same results for refrigerators or heat pumps. They are heat engines that operate in the reverse direction, using work to move heat from a cold place to a hot place. Instead of efficiency, we have the coefficient of performance, which is the ratio of work consumed to heat drawn from the cold place. No irreversible heat pump outperforms a reversible heat pump; and all heat pumps operating between the same temperatures have the same coefficient of performance.

\subsection{Absolute Temperature}

The results of the last section lead to the existence of the absolute temperature scale. To recover it, we consider two reversible heat engines chained together as shown in Figure 4, where as before the quantities of heat and work mentioned are limiting values, unrealized by any heat engine.
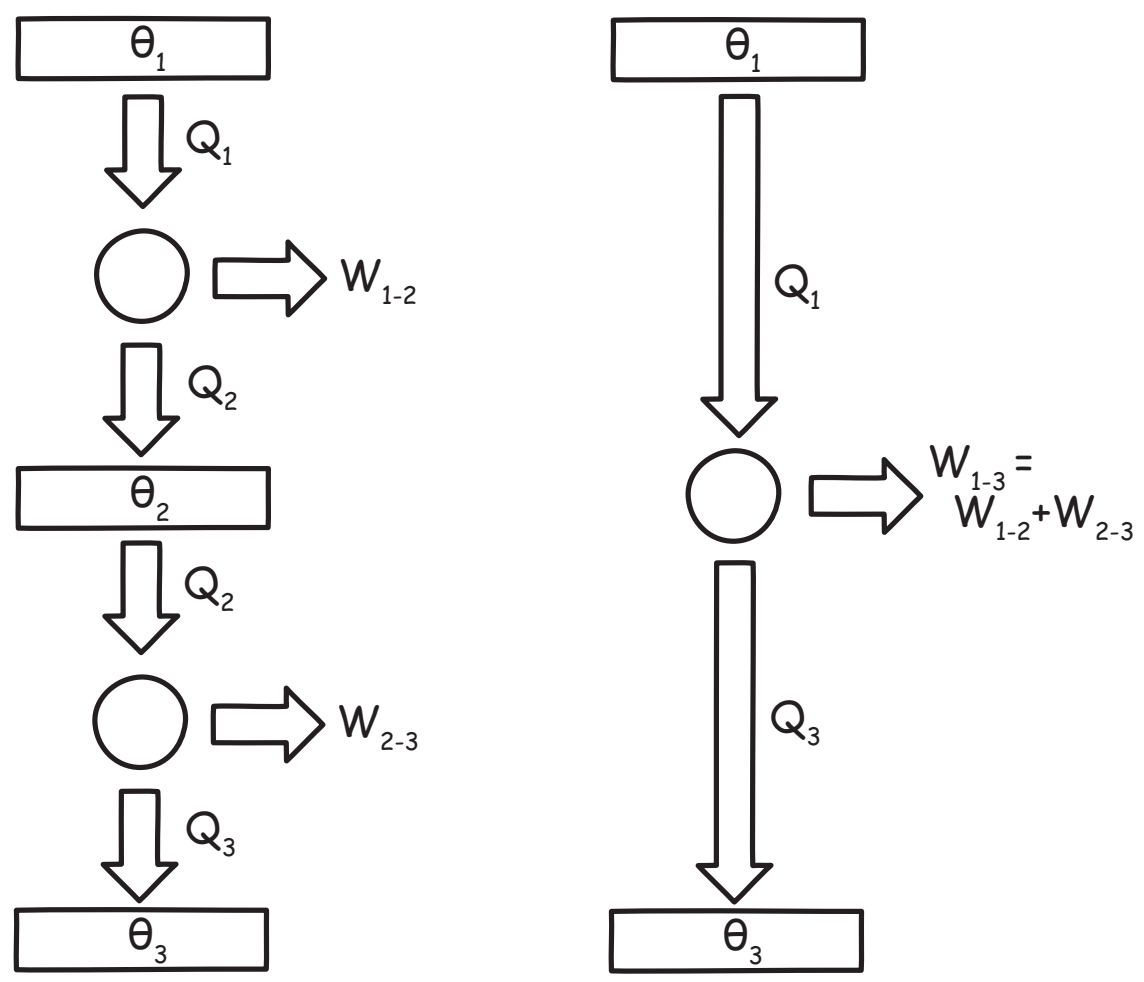

Figure 4. Composition of Heat Engines in the Derivation of Absolute Temperature

The first draws heat $\mathrm{Q}_{1}$ from the highest temperature $\theta_{1}$ and discharges heat $\mathrm{Q}_{2}$ to the intermediate temperature $\theta_{2}$ while creating work $\mathrm{W}_{1-2}$. Temperature is written here as $\theta$ to 
indicate that a definite scale for temperature is not yet assumed. The efficiency $\eta=W_{1-2} / Q_{1}$ of this engine is a function of the temperatures $\theta_{1}$ and $\theta_{2}$ only. It follows that $\mathrm{Q}_{2} / \mathrm{Q}_{1}=$ $\left(\mathrm{Q}_{1}-\mathrm{W}_{1-2}\right) / \mathrm{Q}_{1}$ is also a function of $\theta_{1}$ and $\theta_{2}$ only. Write this function as

$$
\mathrm{f}\left(\theta_{1}, \theta_{2}\right)=\mathrm{Q}_{2} / \mathrm{Q}_{1}
$$

The second heat engine draws heat $\mathrm{Q}_{2}$ from the intermediate temperature $\theta_{2}$ and discharges heat $\mathrm{Q}_{3}$ to the lowest temperature $\theta_{3}$. We have analogously for it

$$
\mathrm{f}\left(\theta_{2}, \theta_{3}\right)=\mathrm{Q}_{3} / \mathrm{Q}_{2}
$$

The two cycles combined, as shown in Figure 4, form a single larger heat engine operating between temperatures $\theta_{1}$ and $\theta_{3}$ with heats $\mathrm{Q}_{1}$ and $\mathrm{Q}_{3}$. We have

$$
\mathrm{f}\left(\theta_{1}, \theta_{3}\right)=\mathrm{Q}_{3} / \mathrm{Q}_{1}
$$

Combining these last three equations we have

$$
\mathrm{f}\left(\theta_{1}, \theta_{3}\right)=\mathrm{Q}_{3} / \mathrm{Q}_{1}=\left(\mathrm{Q}_{3} / \mathrm{Q}_{2}\right) \cdot\left(\mathrm{Q}_{2} / \mathrm{Q}_{1}\right)=\mathrm{f}\left(\theta_{1}, \theta_{2}\right) \cdot \mathrm{f}\left(\theta_{2}, \theta_{3}\right)
$$

It now follows that we can write

$$
f\left(\theta_{1}, \theta_{2}\right)=\frac{f\left(\theta_{1}, \theta_{3}\right)}{f\left(\theta_{2}, \theta_{3}\right)}=\frac{1 / T_{1}}{1 / T_{2}}
$$

where we introduce the absolute temperature $\mathrm{T}$ by definition as 8

$$
T(\theta)=1 / f\left(\theta, \theta_{3}\right)
$$

Finally, combining equations (3) and (4), we have $\mathrm{Q}_{2} / \mathrm{Q}_{1}=\mathrm{T}_{2} / \mathrm{T}_{1}$ so that

$$
\mathrm{Q}_{1} / \mathrm{T}_{1}=\mathrm{Q}_{2} / \mathrm{T}_{2}
$$

\subsection{The Clausius Inequality}

This inequality is an important consequence of the second law of thermodynamics. It asserts that, for any cyclic process, that is any process that returns to its initial state, we have the cyclic intergral

$$
\oint \frac{d Q}{T} \leq 0
$$

\footnotetext{
8 The arbitrary choice of temperature $\theta_{3}$ in the definition affects T only up a multiplicative constant. We can replace it by $\theta_{4}$ to recover

$T^{\prime}(\theta)=1 / f\left(\theta, \theta_{4}\right)=1 / f\left(\theta, \theta_{3}\right) \cdot 1 / f\left(\theta_{3}, \theta_{4}\right)=1 / f\left(\theta_{3}, \theta_{4}\right) . T(\theta)$.
} 
Here I will adapt the compact analysis of Fermi (1937, Ch.4) and Pippard (1966, Ch. 4) to the new characterization of thermodynamically reversible processes.

Consider an irreversible cyclic process undergone by some system $\sigma$. The analysis makes the standard assumption that the process can be approximated arbitrarily well by a sequence of very small isothermal and adiabatic processes. This assumption is not benign. For a process to be isothermal requires that a definite temperature be assigned to the system. If the system is out of equilibrium while partaking in an irreversible process, it is not so clear how this should be done. If the system gains or loses heat, one candidate is the temperature of a large heat reservoir that supplies or accepts the heat. We will not pursue these difficulties here since they are common both to the standard analysis and the variant developed here. The Clausius equality is meaningfully, however, only in so far as this temperature is defined.

The isothermal processes gain or lose heat. We shall assume that this heat is exchanged with a single source system $\sigma_{0}$ that is maintained at a constant temperature $\mathrm{T}_{0}$. For each isothermal process in $\sigma$, the exchange of heat is mediated by a heat engine or heat pump according to whether $\mathrm{T}_{0}$ is greater or lesser than $\mathrm{T}$.

In the standard analysis, this heat engine or heat pump is assumed to be a process that operates reversibly. Hence if an isothermal process gains heat $\Delta \mathrm{Q}$ at temperature $\mathrm{T}$, we have from (5) that these quantities relate to the heat $\Delta \mathrm{Q}_{0}$ drawn from $\sigma_{0}$ at $\mathrm{T}_{0}$ by

$$
\Delta Q_{0}=T_{0} \frac{\Delta Q}{T}
$$

Here, the heat engine or heat pump will be represented by a set of irreversible processes with the limiting, but unrealized behavior of this last relation. That is, we can find an irreversible process that brings the heat drawn from $\sigma_{0}$ to within an arbitrarily small but non-zero $\Delta \varepsilon$ of $\Delta \mathrm{Q}_{0}$ :

$$
\Delta Q_{0}=T_{0} \frac{\Delta Q}{T}+\Delta \varepsilon
$$

The value of $\Delta \varepsilon$ in this last expression will always be negative. ${ }^{9}$ For the adiabatic steps, we will have $\Delta \mathrm{Q}_{0}=0$.

${ }^{9}$ It is simple but tedious to see this negativity. There are six cases overall: the two cases of $\Delta \mathrm{Q}>0$ or $\Delta \mathrm{Q}<0$ paired with the three cases $\mathrm{T}>\mathrm{T}_{0}, \mathrm{~T}<\mathrm{T}_{0}$ and $\mathrm{T}=\mathrm{T}_{0}$. For example, take $\mathrm{T}>\mathrm{T}_{0}$ and $\Delta \mathrm{Q}<0$, so that $\sigma$ loses heat. The heat passes to $\sigma_{0}$ through a heat engine that is slightly less efficient than 
Summing all these quantities of heat $\Delta \mathrm{Q}_{0}$ for the complete cycle in $\sigma$ and taking the limit of arbitrarily small steps in the usual way, we find that the total heat $\mathrm{Q}_{0}$ drawn from the source $\sigma_{0}$ is given by

$$
Q_{0}=T_{0} \oint \frac{d Q}{T}+\delta \quad \text { where } \quad \delta=\oint d \varepsilon
$$

The term $\delta$ is negative, but can be brought arbitrarily close to zero by suitable selection of the engines coupling $\sigma$ and $\sigma_{0}$.

Since the system $\sigma$ is returned to its original state, the sole effect of the cycle is to draw heat $\mathrm{Q}_{0}$ from the source $\sigma_{0}$. By conservation of energy, this heat drawn must go somewhere. The only possibility is that it is transformed into work. Hence, if $\mathrm{Q}_{0}>0$, we have a full conversion of heat into work, in violation of the Thomson form of the second law of thermodynamics. Hence the cycle either must have no net effect or must degrade work into heat that is passed to $\sigma_{0}$. That is, $\mathrm{Q}_{0} \leq 0$, so that

$$
T_{0} \oint \frac{d Q}{T} \leq-\delta
$$

Since $\oint \frac{d Q}{T}$ is a fixed magnitude specified by the particular process in $\sigma$, but the positive $-\delta$ can be made arbitrarily small, we must have the Clausius inequality (6) 10

$$
\oint \frac{d Q}{T} \leq 0
$$

\subsection{The Path Independence of Thermodynamic Entropy}

Clausius (1865) introduced thermodynamic entropy S by means of the definition:

$$
\Delta S=S_{2}-S_{1}=\int_{1}^{2} \frac{d Q_{r e v}}{T}
$$

the reversible efficiency. As a result slightly more heat will pass to $\sigma_{0}$ than reversible efficiency indicates. Since this heat passed $\Delta \mathrm{Q}_{0}$ is negative, it will be more negative by an added amount $\Delta \varepsilon$ that is also negative.

10 For irreversible processes, this should be a strict inequality, but I do not see how to recover it from the analysis given here. 
where $\Delta \mathrm{S}$ is the change of entropy between two states 1 and 2 and $\mathrm{Q}_{\mathrm{rev}}$ is the heat passed to the system while at temperature $\mathrm{T}$ in the course of a thermodynamically reversible process that connects the two states.

For entropy to be a function of the equilibrium state of the system, it is essential that the entropy difference $\Delta S$ be the same no matter which reversible process is used to compute the integral. The inferences that establish this path independence are common in thermodynamics texts and can be found in various forms in Clausius (1879, Ch. IV), Poynting and Thomson (1920, Ch. XVII), Fermi (1937, Ch.4) and Pippard (1966, Ch. 4).

Employing the conception of Section 2.2, consider a cyclic reversible process; that is, one whose set of equilibrium states form a closed curve in the equilibrium state space as shown in Figure 5. We apply the Clausius inequality (6) to the forward and to the reversed set of irreversible processes that comprise the reversible processes. As the driving forces go to zero, the limiting values of the two resulting cyclic intergrals are indicated by subscripts $\mathrm{f}$ and $\mathrm{r}$ and must satisfy relations:

$$
\oint \frac{d Q_{f}}{T} \leq 0 \quad \oint \frac{d Q_{r}}{T} \leq 0 \quad \oint \frac{d Q_{f}}{T}=-\oint \frac{d Q_{r}}{T}
$$

The first two inequalities result from the Clausius inequality and the third equality from conditions (e) and (f) Section 2.2. These relations can only be satisfied if:

$$
\oint \frac{d Q_{f}}{T}=\oint \frac{d Q_{r}}{T}=\oint \frac{d Q_{r e v}}{T}=0
$$

where $\mathrm{Q}_{\mathrm{rev}}$ is the heat associated with a reversible process. 


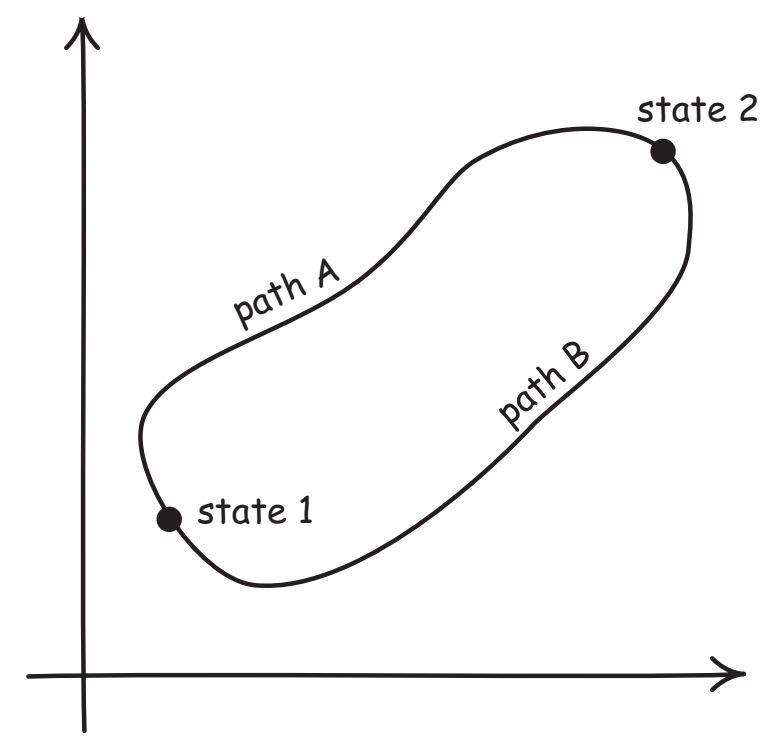

Figure 5. Closed curve in equilibrium state space.

Standard arguments now establish that entropy, as defined by the Clausius formula (7), is a state function. Choose any two states 1 and 2 through which a closed curve in equilibrium state space passes. The two states can be connected by either path A or path B of Figure 5. For the complete cycle we have

$$
0=\oint \frac{d Q_{\text {rev }}}{T}=\int_{p_{\text {path }} A}^{2} \frac{d Q_{\text {rev }}}{T}+\int_{\text {path } B}^{1} \frac{d Q_{\text {rev }}}{T}=\int_{1_{\text {path }} A}^{2} \frac{d Q_{\text {rev }}}{T}-\int_{p_{\text {path } B}}^{2} \frac{d Q_{\text {rev }}}{T}
$$

The last equality follows since the quantities of heat change sign on reversal of the path direction. It now follows that

$$
\int_{p_{\text {path } A}^{2}}^{2} \frac{d Q_{\text {rev }}}{T}=\int_{1 \text { path } B}^{2} \frac{d Q_{\text {rev }}}{T}
$$

so that the quantity $\Delta \mathrm{S}$ computed in formula (7) is independent of the path taken from state 1 to state 2 .

Since $\mathrm{dQ}_{\mathrm{rev}}=\mathrm{dU}-\mathrm{dW}$ by the first law (2) and $\mathrm{dW}=\sum_{\mathrm{i}} \mathrm{X}_{\mathrm{i}} \mathrm{dx}_{\mathrm{i}}$ by (1), the magnitudes of entropy changes $\Delta S$ are determinable from the state variables by means of Clausius' formula (7). Hence the entropy $S_{2}=S_{1}+\Delta S$ of any state is now fixed up to the selection of the state 1 as a universal reference state.

The Clausius inequality now enables us to interpret the significance of entropy for irreversible processes. Consider an irreversible process that initiates arbitrarily close to state 1 
and passes in any way to a state arbitrarily close to state 2 ; and then returns to state 1 , with a process whose driving forces approach zero such that its states everywhere come arbitrarily close to path $\mathrm{B}$ of Figure 5 . We have from the Clausius inequality that ${ }^{11}$

$$
0 \geq \oint \frac{d Q}{T}=\int_{1, \text { any path }}^{2} \frac{d Q}{T}+\int_{2, \text { path } B}^{1} \frac{d Q}{T}
$$

The second integral is equal to $-\left(\mathrm{S}_{2}-\mathrm{S}_{1}\right)$. That is, we have

$$
S_{2}-S_{1} \geq \int_{1, \text { any path }}^{2} \frac{d Q}{T}
$$

The system in which states 1 and 2 arise may be of arbitrary complexity and may exchange heat and work between its parts in many ways. If we now suppose, however, that the system exchanges no heat with its environment, this last inequality reduces to

$$
\mathrm{S}_{2} \geq \mathrm{S}_{1}
$$

We now have a major result: the entropy of an isolated system can never decease.

Another consequence of comparable importance applies to the case of a system undergoing any isothermal process at temperature $\mathrm{T}$, while exchanging heat but not work with its surroundings. For such a process, inequality (8) becomes $\Delta S=\left(S_{2}-S_{1}\right) \geq Q / T$. Since no work is exchanged with the surroundings the heat gained $\mathrm{Q}$ equals the change of internal energy $\Delta \mathrm{U}$. Rearranging, we have

$$
\Delta \mathrm{F}=\Delta(\mathrm{U}-\mathrm{TS}) \leq 0
$$

That is, the free energy $F=U$ - TS of such a system cannot increase. The results of (6), (9) and (10) are not the strongest, since the equality in each cannot be realized by any real, that is, irreversible process.

We have now rederived some of the principal results of thermodynamics using a characterization of thermodynamically reversible processes free of overt internal contradictions. The exercise could continue, but the results are a foregone conclusion. It turns out that axiomatic developments of thermodynamics in the tradition of Carathéodory do not require the attribution of further or different properties to thermodynamically reversible processes. Zemansky (1996;

11 The exact expressions result here from exercising the freedom to bring the process states arbitrarily close to states 1,2 and those along path B. 
1968, $\S \S 8-7$ to 8-10, 9-1) has shown that Carathéodory’s analysis can be embedded within a traditional analysis similar to the one just given, but perhaps without the mathematical erudition.

\section{Molecular Scale Systems: When the Difference Matters}

The account of thermodynamically reversible processes of Section 2.2 includes an existence assumption. Whether it obtains depends on the physics governing the dynamical processes. It most likely does not fail as long as we neglect the molecular constitution of matter. More precisely, it will hold most likely in a fictitious world in which ordinary thermodynamics holds exactly at all scales. In this fictitious world, temperature and heat are not explicated statistically, but are primitive properties of matter.

The existence assumption will fail, however, once we allow that real matter has a molecular character and we consider molecular scale systems in which thermal fluctuations play a prominent role. Completion of any process at molecular scales requires that these fluctuations can be overcome. They are overcome by net driving forces that cannot be made arbitrarily small, in contradiction with condition (c). Even with imbalanced driving forces, no process is assured completion. We can at best have some definite probability of completion. If, for example, a temperature difference drives heat from one body to a second, there is always a small probability that a thermal fluctuation will return the heat and undo the process.

In work elsewhere (Norton, 2013, Part 2; 2013a; forthcoming), I have derived the general results governing this effect. Consider an isolated process. If its initial and final states have entropies $S_{1}$ and $S_{2}$, then the system will fluctuate between the two states with probabilities $p_{1}$ and $\mathrm{p}_{2}$, where

$$
\frac{p_{2}}{p_{1}}=\exp \left(\frac{S_{2}-S_{1}}{k}\right)
$$

for k Boltzmann's constant. There is an analogous result for an isothermal process that exchanges heat but not work with its environment - this is the type of process considered routinely in the thermodynamics of computation. If the initial and final states have free energies $\mathrm{F}_{1}$ and $\mathrm{F}_{2}$, then the system will fluctuate between the states with probabilities $\mathrm{p}_{1}$ and $\mathrm{p}_{2}$, where

$$
\frac{p_{2}}{p_{1}}=\exp \left(-\frac{F_{2}-F_{1}}{k T}\right)
$$


Thus probabilistic completion of the processes can only be assured if they are both dissipative. The probability ratio $\mathrm{p}_{2} / \mathrm{p}_{1}=0.95$ assures only a modest chance of completion, but it requires entropy creation of $\Delta \mathrm{S}=\mathrm{S}_{2}-\mathrm{S}_{1}=3 \mathrm{k}$ in the first case and a correspondingly dissipative reduction in free energy $\Delta \mathrm{F}=\mathrm{F}_{2}-\mathrm{F}_{1}=-3 \mathrm{kT}$.

In sum, on molecular scales, thermodynamically reversible processes cannot be realized in the sense that no completing process can be brought arbitrarily close to a sequence of equilibrium states. Thermodynamic reasoning is still possible for systems at molecular scales. However an uncritical supposition of thermodynamically reversible processes at this scale can lead to quite unfounded results. In a series of papers developed and summarized in Norton (2013), I have sought to demonstrate that just this uncritical supposition is part of the accumulation of theoretical failures comprising what is called the "thermodynamics of computation."

\section{PART II. A Critical Historical Survey of Accounts of Thermodynamically Reversible Processes}

\section{A Mechanical Analog}

One would not expect that the mechanics of ordinary machines could provide a model of thermodynamically reversible processes. For in mechanics, the least dissipative processes are those governed by a conservative Hamiltonian. No matter how violent or rapid its motion, a bouncing ball dissipates no energy as long as its bounce is fully elastic. In thermodynamics, however, the least dissipative processes are thermodynamically reversible and they proceed arbitrarily slowly without the violence of greatly imbalanced forces.

This difference would not have been so apparent in Carnot's time. For the less refined machines of his time operated dissipatively, far from this elastic ideal. Their parts collided inelastically and converted work to heat. Efforts to improve their efficiency faced problems similar to those faced by heat engines. Just as in the thermodynamic case, such machines are made more efficient by minimizing all imbalances of forces and thereby slowing them down to an arbitrarily great extent. 
Sadi Carnot's father Lazare Carnot $(1786,1808)$ developed a lengthy account of the efficiency of these machines. Lazare (1808, 30, pp. 11-12) concluded for them:

...in order to make them produce the greatest possible effect, it must necessarily happen that there be no percussion, i.e. the movement should always change by imperceptible degrees;...

The percussions are dissipative, inelastic collisions. The greatest effect is produced by minimizing the imbalance of forces that produces them. This is the analog of the condition that leads to thermodynamically reversible processes.

Lazare's analysis included (1808, 31, pp. 300-301) "hydraulic machines moved by a current of water"--that is, water wheels. Once optimized according to two conditions he specified, Lazare concluded that

... the form of the machine would be of little consequence; for a hydraulic machine which will fulfil these two conditions will always produce the greatest possible effect ...

The parallel to the son's, Sadi Carnot's (1824), results in unmistakable. He concluded that a reversible heat engine is the most efficient. It delivers that efficiency independently of the details of its construction, including notably whether the working fluid is steam or air.

In his memoir, Sadi Carnot (1824, pp. 60-61) compared the fall of water in a water wheel to the passage of caloric from hot to cold. The analogy to the least percussive processes of Lazare Carnot is as important and possibly more so, since those processes are the analogs of thermodynamically reversible process, which are the core of Carnot's and later thermodynamic theory.

\section{What Thermodynamically Reversible Processes are Said to Be (and why they are not quite so)}

My goal in this section is to inventory the characterizations of thermodynamically reversible processes given in the literature. What will result is not a catalog of mutually incompatible definitions or characterizations. For the characterizations are mostly mutually interdependent and that fact is widely recognized. If one decides, for example, that a thermodynamically reversible process is one that is very slightly removed from equilibrium, it 
will follow that it is a process that can be reversed with slight changes of the driving forces; and the converse also follows. Rather the inventory merely seeks to individuate the characterizations with some indication of who in the literature uses each to introduce or even define thermodynamically reversible processes.

Since the notion of thermodynamic reversibility has been present since the beginning of thermodynamics nearly 200 years ago in Carnot (1824), the pertinent literature is enormous and this inventory must be quite far from exhaustive. ${ }^{12}$ I apologize to readers whose favorite characterization has been omitted. 13

The inventory, however, is sufficient to establish that all is not well in the literature. With the sole exception of Duhem's account discussed in Section 2.3, all of the characterizations I survey are inadequate. Either they are incomplete or they include provisions that contradict one another, so that the characterization overall is a contradiction when read literally.

This last assertion is my summary conclusion. I do not conclude that the notion of a thermodynamically reversible process is unsalvageable. That salvage was the work of Section 2. I do conclude that, in their eagerness to keep things simple, authors writing on thermodynamically reversible processes go too far in the simplifications and commonly end up with literal nonsense. That is, they give descriptions that are internally contradiction and thus cannot have a proper meaning or sense.

For further details on different senses of reversibility, how they connect and enter into thermodynamic theorizing, see Uffink $(2001,2006)$. One result of his investigation is that is that the German language literature uses two words for reversible-umkehbar and reversibel-where English writers use one. Taking Planck's (1897) treatise as authoritative and highly influential in spite of its weaknesses on the subject, Uffink $(2001, \S 7)$ finds Planck to use umkehbar to mean quasi-static and reversibel to mean recoverable.

12 A Google search in June 2015 identified over 15,000 books with the word "thermodynamics" in the title.

13 One familiar characterization, such as Landau and Lifshitz (1970, p.32), is that thermodynamically reversible processes are ones in which the total entropy of the system and surroundings remain unchanged. I exclude it since the standard Clausius definition of entropy itself requires the prior notion of thermodynamically reversible processes. 


\subsection{Existential Supposition}

The simplest way that thermodynamically reversible processes enter is by supposition. A process is described and, in passing, it is declared that the process could have happened in the reverse direction, with all quantities of heat transferred and work done reversing their signs. This is how the notion enters in Carnot's original memoire. After describing a heat engine that operates on what we now call a Carnot steam cycle, he declares (1824, p. 54) "The operations which we have just described might be have been performed in an inverse direction." Carnot recognized that a process admitting such reverses might be troublesome, but the worry is confined to various remarks in passing, as will be discussed in Sections 7.2 and 7.4 below.

Subsequent authors in this early period give readers no explicit indication of the complications of realizing a thermodynamically reversible process. Clapeyron's (1837) revival of Carnot's dormant memoire merely notes for a Carnot cycle: "The inverse operation is equally possible..." (p. 351). Clausius and Thomson, in their writings from around 1850 that lay the foundations of the modern theory, proceed likewise. Clausius $(1851$, p. 103) simply describes the reversal of a cyclic process. Thomson $(1849$, p.139) alerts the reader to the importance of a reversible heat engine with an italicized definition, but gives no indication of the difficulties in realizing one:

A perfect thermodynamic engine is such that, whatever amount of mechanical effect it can derive from a certain thermal agency, if an equal amount be spent in working backwards, an equal reverse thermal effect will be produced.

Thomson (1852, p. 12) introduces reversible heat engines as a supposition within a consequence of the second law of thermodynamics:

Prop. II (Carnot and Clausius) - If an engine be such that, when it is worked backwards, the physical and mechanical agencies in every part of its motions are all reversed, it produces as much mechanical effect as can be produced by any thermodynamic engine, with the same temperatures of source and refrigerator, from a given quantity of heat.

Similarly Tait (1877, p. 44) and Roengten (1880, Ch. VI) declare the reversibility of a cycle without apology. This tradition of existential supposition persisted. Goodenough (1911, p. 47) asserts: 
35. Reversibility. - The processes described in thermodynamics are either reversible or irreversible. A process is said to be reversible when the following conditions are fulfilled :

1. When the direction of the process is reversed, the system taking part in the process can assume in inverse order the states traversed in the direct process.

2. The external actions are the same for the direct and reversed processes or differ by an infinitesimal amount only.

3. Not only the system undergoing the change but all connected systems can be restored to initial conditions.

It is only two pages later that Goodenough (p. 49) admits in passing that such a process would require temperature differences that are "indefinitely small" so that

Strictly speaking, there are no reversible changes in nature. We must consider reversibility as an ideal limiting condition that may be approached but not actually attained when the processes are conducted very slowly.

\section{The problem}

Mere supposition of the existence of a process is inadequate if the process supposed contradicts other suppositions of the theory. Other than for purposes of reductio, we would not allow constructions in thermodynamics that depend upon the existence of a perpetual motion machine. Since thermodynamically reversible processes contradict the requirement that change needs unbalanced driving forces, why should we treat them differently?

\subsection{Driving Forces Differ Insensibly from Zero}

These early writers did sometimes introduce remarks later to soften their impossible demand for a system to be both to be in equilibrium and out of equilibrium at the same time. They concern the driving forces that move a system away from equilibrium, such as non-zero temperature differences and imbalances of mechanical forces. These differences are required to be close enough to zero for the difference not to matter. Here is Carnot's $(1824$, p. 58) version

In reality the operation cannot proceed exactly as we have assumed. To determine the passage of caloric from one body to another, it is necessary that there should be an excess of temperature in the first, but this excess may be supposed as slight as 
we please. We can regard it as insensible in theory, without thereby destroying the exactness of the arguments.

Clausius writing later in the 1865 paper that introduced the notion of entropy addressed the concern that no work can be done in a reversible process in which force and counterforce balance exactly (p. 357):

However, since the difference between them can be made arbitrarily small, one can consider the case in which there is absolute equality as the boundary case that, even if it is never attainable in reality, is still to be considered as possible theoretically. In his later Mechanical Theory of Heat, Clausius (1879) makes similar remarks concerning reversible heat transfers, which can only occur between bodies at the same temperature (p. 106):

This condition cannot indeed be exactly fulfilled, since between equal temperatures there can in general be no passage of heat whatever; but we may at least assume it to be nearly so fulfilled that the small remaining differences of temperature may be neglected.

Eventually, however, it became clear that the delicate statement of the delicate conditions needed for reversibility required more explicit treatment. Poynting and Thomson's (“J. J.”, not William) (1920) ubiquitous textbook Heat devoted nearly a full page to specifying these conditions for the operation of a Carnot cycle. Under the heading "Conditions for Reversible Working," the conditions include (p. 264):

...the working substance shall never differ sensibly in temperature from the bodies to which it is giving or from which it is receiving heat...

... the pressure exerted by the working substance on the piston shall be sensibly equal to the load...

...the machinery moves without friction...

We shall see below in Section 2.8 that Carathéodory's (1909, p. 366) initial introduction of his notion of quasi-static process also called upon differences that fall "beneath the limit of observation." 


\section{The Problem 14}

If the deviation from equilibrium is so unimportant, it should be dropped. Of course it cannot, for then there would be no change in time. It is a difference that makes a difference. This characterization tries to have two propositions true at the same time: The states of the process are in equilibrium; and the states are not in equilibrium. The problem is the law of the excluded middle of logic. Either a proposition is true; or its negation is true; but not both. There is no third option.

This is an unforgiving assessment. No doubt these authors did not really intend to assign contradictory properties to reversible processes. Presumably they expected that a more explicit analysis, paying due attention to notions like approximation and idealization, would eliminate the appearance of a contradiction. Perhaps they would even assent to the account of Sections 2, 3 and 4 above. My point, however, is that they did not give this account. They leave a central term in their expositions poorly defined.

\subsection{Processes Reversed by Very Small Changes of Driving Force}

These early writers agreed that thermodynamically reversible processes must have net driving forces that are little different from zero and imperceptibly, insensibly and extremely so. However, while they surely knew, they do not make explicit what this condition has to do with reversibility. That connection produced a variant form of the characterization that is, I believe, close to the right way to conceive of thermodynamically reversible processes.

Loosely, the idea is that a thermodynamically reversible process is everywhere so close to a perfect balance of driving forces, that a slight disturbance to them can reverse the direction of the process. That is the origin of the reversibility.

Maxwell, in his Theory of Heat, gives an early example. In describing the operation of a Carnot cycle engine, he notes (pp. 149-50) that the engine working substance exchanges heat with a body that differs from it "extremely little" in temperature and, by slowing the operation, it can be made "as small as we please." Then, to sustain the reversed motion, “... an exceedingly small alteration of the temperature will be sufficient to reverse the flow of heat, if the motion is

14 Full disclosure: Norton (1998, p. 6-22-23) writes "An individual process is reversible if it is only minutely away from equilibrium." 
slow enough." His analysis employs everywhere very small deviations from equilibrium, quite in the manner of the analysis of Section 4 above. However eventually Maxwell cannot resist taking the paradoxical limit (p. 150):

But by working the engine sufficiently slowly these differences may be reduced within any limits we please to assign, so that for theoretical purposes we may regard Carnot's engine as strictly reversible.

This has become the standard characterization of reversibility in some 20th century texts. Pippard's (1966) widely read Elements of Classical Thermodynamics makes it the definition (p. 22, emphasis in original):

A reversible process is defined as one which may be exactly reversed by an infinitesimal change in the external conditions.

This characterization then leads to the conclusion that a reversibly expanding fluid may be treated as if in equilibrium, for Pippard concludes (p. 23):

To sum up, if a fluid be caused to undergo an infinitesimal change reversibly, it is legitimate to apply the first law to the change in the form

$$
\mathrm{dU}=\mathrm{dQ}-\mathrm{PdV}
$$

Pippard had argued earlier (p. 21) that the work term PdV of the first law only has meaning if the expansion is such that the fluid "pass[es] through all intermediate states of equilibrium."

Van Ness (1969) describes how a gas can be expanded reversibly by counterbalancing the gas pressure with the constantly adjusted weight of a pile of sand (p. 22, emphasis in original):

This imaginary process is called reversible because at any point it could be turned around and made to go the other way simply by replacing the infinitesimal grains of sand on the piston. Only one additional infinitesimal grain of sand would be needed to start the reverse process.

Thus the states through which the system passes during a reversible process are for all practical purposes equilibrium states, or more precisely are never removed more than differentially from equilibrium states. 
One also finds the characterization in the journal literature. MacDonald (1995) offers this summary definition (p. 1122, emphasis in original):

In a quasistatic process, the thermodynamic system moves through a linear continuum of equilibrium states... A quasistatic process is carried out reversibly if a slight change in the forces (thermal and mechanical) driving the process can reverse it. (Slow heating of water by a resistor is an example of a quasistatic irreversible process.)

Finally this is the account offered by Lieb and Yngvason (1998). Writing of an adiabatic expansion of a gas from state $\mathrm{X}$ to state $\mathrm{Y}$, they note (p. 573):

On the other hand, we could let the piston expand very, very slowly by letting it raise a carefully calibrated weight. No other machinery is involved. In this case, we can reverse the process (to within an arbitrarily good accuracy) by adding a tiny bit to the weight, which will then slowly push the piston back. Thus, we could have (in principle, at least) both ${ }^{[15]} \mathrm{X} \prec \mathrm{Y}$ and $\mathrm{Y} \prec \mathrm{X}$, and we would call such a process a reversible adiabatic process.

The delicacy of the "in principle" qualification is made clearer in Lieb and Yngvason (1999, p. 17):

When $\mathrm{X} \prec \mathrm{Y}$ and also $\mathrm{Y} \prec \mathrm{X}$, then the state change can only be realized in an idealized sense, for it will take infinitely long time to achieve it in the manner described.

\section{The problem}

In practice, this alternative characterization of reversal by imperceptibly small changes in driving forces reduces to the earlier characterization of Section 7.2 where deviations from equilibrium are deemed too small to matter. Thus it faces the same problems mentioned above. We cannot have states $\mathrm{X}$ and $\mathrm{Y}$ intertransformable according to both $\mathrm{X} \prec \mathrm{Y}$ and $\mathrm{Y} \prec \mathrm{X}$, for example, for that would violate the principle that bodies only move when there is a net, non-zero force applied to them.

15 For " $\mathrm{X} \prec \mathrm{Y}$ " read "there is an adiabatic process that transforms $\mathrm{X}$ into $\mathrm{Y}$." 


\subsection{Processes that are Infinitesimally Removed from Equilibrium}

The last two characterizations depend upon driving forces being so small that their difference from zero is too small to matter. This behavior is reminiscent of the use of infinitesimals in the calculus and many (including Pippard above) have adopted that language in their characterization of thermodynamically reversible processes. Here, for example, is Fermi's (1937) version: (p. 4, emphasis in original)

A transformation is said to be reversible when the successive states of the transformation differ by infinitesimals from equilibrium states.

Earlier, Lewis and Randall (1923) had combined this condition with several others, strictly incompatible with it. The "ideal or reversible process" they say (p. 112) is one in which ...all sources of dissipation are eliminated. It is to be regarded as a limit of actually realizable processes.

Let us imagine a process so conducted that at every state an infinitesimal change in the external conditions would cause a reversal in the direction of the process; or, in other words, that every step is characterized by a state of balance. Evidently a system which has undergone such a process can be restored to its initial state without more than infinitesimal changes in external systems. It is in this sense that such an imaginary process is called reversible.

Porter (1931), in describing Carnot's innovations, also employs the notion of an infinitesimal change: (p. 14, emphasis in original)

...the cycle of change to be a reversible one; by which is meant that by making only an infinitesimal change in the temperatures and pressures of the surroundings the cycle may be traversed either in a clockwise or an anticlockwise sense: in other words, at each instant the substance must be indefinitely near to an equilibrium state both as regards pressure and of heat flow. This, of course, describes an unattainable limit, because it would require an infinite time to effect any change.

Porter later (p. 20) indicates the changes are of the same type as "virtual displacements made in mechanics in the calculation of virtual work."

The appeal of the connection is easy to see. Infinitesimals in the calculus are, loosely speaking, differences that are smaller than any ordinary magnitude, but nonetheless not zero. So 
they do bear some resemblance to what is needed in characterizing thermodynamically reversible processes. They commonly arise in the taking of derivatives. A small, finite change $\Delta \mathrm{x}$ in the argument of the function $\sin (\mathrm{x})$ yields a small change in the function $\Delta \sin (\mathrm{x})$ that satisfies the equality only approximately

$$
\Delta \sin (\mathrm{x}) \approx \cos (\mathrm{x}) \Delta \mathrm{x}
$$

It is standard to replace this approximate equality by an exact equality by replacing the small differences with the infinitesimals $\mathrm{d} \sin (\mathrm{x})$ and $\mathrm{dx}$ :

$$
\mathrm{d} \sin (\mathrm{x})=\cos (\mathrm{x}) \mathrm{dx}
$$

Division by $\mathrm{dx}$, which would be impossible if $\mathrm{dx}$ were strictly zero, then gives us the exact derivative

$$
\frac{d \sin (x)}{d x}=\cos (x)
$$

It is customary to treat the infinitesimals as quantities greater than zero but smaller than any positive real since that makes the manipulations the same as those of ordinary algebra. However that treatment is, strictly speaking, nonsense since there are no real numbers meeting this description. ${ }^{16}$

What makes these manipulations legitimate is that there is a second reading for these equations. In it, the symbol "d" no longer represents an infinitesimal difference. Rather it is the derivative operator $\mathrm{d} / \mathrm{dx}$, defined by the usual limiting procedure

$$
\frac{d f(x)}{d x}=\operatorname{Lim}_{\Delta x \rightarrow 0} \frac{f(x+\Delta x)-f(x)}{(x+\Delta x)-x}
$$

If precision and meaning becomes the issue, we simply revert to this precise interpretation.

This analogy to the differential calculus extends back to the beginning of thermodynamics. Carnot (1824, p. 53) is clearly alluding to it when he writes:

16 Atkins (2010, p. 27, emphasis in original) defines a reversible process as "one that is reversed by an infinitesimal modification of the conditions in the surroundings" and adds "The key word is infinitesimal." An infinitesimal is treated as the smallest non-zero displacement. For (p. 28) a reversible expansion is reversed "...if at any stage the external pressure is increased even infinitesimally, then the piston will move in rather than out." 
We may perhaps wonder here that the body B being at the same temperature as the steam is able to condense it. Doubtless this is not strictly possible, but the slightest difference of temperature will determine the condensation, which suffices to establish the justice of our reasoning. It is thus that, in the differential calculus, it is sufficient that we can conceive the neglected quantities indefinitely reducible in proportion to the quantities retained in the equations, to make certain of the exact result.

\section{The problem}

Infinitesimals can be used in ordinary differential calculus in spite of their internal contradictions, since they are surrogates for differential operators free of manifest contradiction. We are, it seems, supposed to presume that since infinitesimals can be used in the calculus, they are also licit in the new setting. These authors in thermodynamics, however, provide no account of unproblematic surrogates for their infinitesimals. Without such an account, the contradictions internal to the account remain. 17

\subsection{Infinitely Slow Processes}

The complement to the infinitesimally small driving force is the infinitely slow process that takes infinitely long to complete. This is a standard characterization. Perhaps the best known is Planck's (1887, pp. 49-50):

Special theoretical importance must be attached to those thermodynamical

processes which progress infinitely slowly, and which, therefore, consist of a succession of states of equilibrium. Strictly speaking, this expression is vague, since a process presupposes changes, and, therefore, disturbances of equilibrium. But where the time taken is immaterial, and the result of the process alone of

17 An interesting possibility is that non-standard analysis might provide an account of these infinitesmals free of manifest contradiction. I have found no such account in the literature and expect the reconstruction would be quite complicated. It would include various hyperreal infinities for the times of the processes and a means of assigning thermodynamic quantities to states infinitesimally removed from equilibrium. 
consequence, these disturbances may be made as small as we please, certainly very small in comparison with the other quantities which characterize the state of the system under observation.

Planck continues to describe how infinite slowness is achieved in processes. They have external pressures that are, for example, "just a trifle" greater than the system gas pressure and heat reservoirs with temperatures "of slightly higher or lower temperature." Then a limit to infinite slowness is taken.

These infinitely slow processes are connected with reversible processes by a familiar argument (p. 51, emphasis in original)

The value of this method of viewing the process lies in the fact that we may imagine each infinitely slow process to be carried out also in the opposite direction. If a process consist of a succession of states of equilibrium with the exception of very small changes, then evidently a suitable change, quite as small, is sufficient to reverse the process. This small change will vanish when we pass over to the limiting case of the infinitely slow process, for a definite result always contains a quite definite error, and if this error be smaller than any quantity, however small, it must be zero.

Unfortunately, this characterization can be quite misleading, unless one is paying attention to the details. Mere infinite slowness is not enough to deliver a thermodynamically reversible process. Counterexamples appear repeatedly in the literature.

The simplest is just a vessel containing a gas under pressure that leaks extremely slowly in a larger, evacuated chamber through a tiny hole. The process can be made arbitrarily slow by making the hole arbitrarily small. Then, during the process, the states of the gas in the vessel and in the larger chamber can be brought as close to equilibrium as we like. A gas expanding while doing no work, no matter how slowly, is a paradigm example of an irreversible process.

A variant consists of a gas contained within a succession of very many, closely spaced barriers or membranes. They are slid away or punctured one by one from the inside, allowing the gas to expand slightly each time, while doing no work, to achieve a new equilibrium at each stage. In such a process, an ideal gas will trace out the curve (12) below of equilibrium states, while once again undergoing an irreversible expansion. See Kestin (1979, pp. 130-31); Uffink, (2001, p. 344). 
Many variants are possible. Sommerfeld, $(1962$, p. 17) describes an electrically charged capacitor whose stored energy is converted irreversibly to heat by discharge through a resistor. The process can be slowed arbitrarily by employing an arbitrarily large resistance, yet the process remains irreversible.

The requirement of infiniteness slowness must be supplemented by a second condition already implicit in Planck's examples: the infinite slowness arises because the forces driving the process forward are brought arbitrarily close to perfect balance. As a result, the capacity of gas pressure or higher temperature to do work is not lost. This condition fails in the irreversible processes just considered.

A more careful characterization will include this further condition. For example, Sommerfeld (1956, p. 19) writes:

Reversible processes are not, in fact, processes at all, they are sequences of states of equilibrium. The processes which we encounter in real life are always irreversible processes, processes during which disturbed equilibria are being equalized. Instead of using the term "reversible process" we can also speak of infinitely slow, quasistatic processes during which the system's capacity for performing work is fully utilized and no energy is dissipated.

\section{The problem}

In thermodynamics, an infinitely slow process is a process that is slowed arbitrarily in all its stages. Considering seriously the completion of such processes invites trouble. How are we to chain two such processes together, so that the second begins when the first completes? The first never completes, so the second never begins. Similarly, how are we to understand that the first process can complete, when that completion cannot happen until an initial proper part of process has completed. Yet completing that proper part will also require an infinity of time first to elapse.

Fortunately, we need not linger over such puzzles. These infinitely slow processes are just process in which no changes at all occur. They are frozen for all time in their initial states. To imagine otherwise is to imagine that there is some moment in time, infinitely far in the future, at which the process is completed. There is no such moment of time. Every moment of future time comes immediately after some finite time has elapsed and, by supposition, no change has occurred after each finite time. Since there is no moment of time infinitely in the future, talk of 
completion of an infinitely slowed process is literally nonsense: speech whose words do not refer to anything.

It is remarkable that authors who pride themselves on precision of analysis are willing to recognize these elementary facts but dismiss them with inadequate deflections. Buchdahl (1966, p. 11) writes:

The requirement that any finite quasi-static transition will require an infinite interval of time must not be taken too seriously. What effectively constitutes a quasi-static transition in practice can ultimately be decided only by an appeal to relaxation times ... Here, as in any theory involving the consideration of conditions unrealizable in practice, one just has to be 'reasonable' in the interpretation of idealizations which have been introduced: granted that such idealizations do not come into conflict with some basic natural law.

Presumably being "reasonable" is an invitation to drop strict standards of rigor. The encouragements to drop the standards are inadequate. A study of relaxation times will only tell us how long a process requires to slow to some nominated degree, while never actually stopping. (See equations (15) and (16) below.) Moreover the idealization of infinite slowness does conflict with basic natural laws: in thermal physics, heat cannot be transferred without a non-zero temperature difference; in dynamics, a body cannot be moved without a non-zero force; in spacetime geometry, there is no real event at temporal infinity where these processes are completed.

Even authors of axiomatic systems dismiss the problems. As we saw, Lieb and Yngvason (1999, p.17) develop a precise axiom system in which two thermal states can be accessed from each other in what would be a reversible process, even though "it can only be realized in an idealized sense, for it will take infinitely long time to achieve it..."

\subsection{Process in which the Initial State can be Restored}

In a thermodynamically reversible process, a certain quantity of heat is gained by the system and a certain amount of work is done by it. This heat is transferred from some system of heat reservoirs; and the work is captured by mechanical devices, typically idealized as the raising of weights. When the reversed process is carried out, the same magnitude of heat and work are exchanged, but they pass in the opposite direction. If heat is gained in the forward direction, it is 
lost in the reversed direction. If the system does net work on the surroundings in the forward direction, net work is done on it in the reverse direction. As a result, if a thermodynamically reversible process is then immediately followed by its reversal, the heat reservoirs and mechanical devices with which it interacted can be restored to their original states, as will the system itself.

This property of full restoration has been used as a distinctive characteristic of thermodynamic processes. Mcaulay (1913, p. 16) expresses it as:

A reversible process is one which can be retraced, so that the substance passes through the same states as in the direct process, but in the reverse order; the amounts of heat received and of work done in each step being the same as in the direct process, but with opposite signs; heat being parted with where in the direct process it was received, and work being done upon the substance by some external agency where in the direct process it did work.

Zemansky (1968, pp. 191-92, emphasis in original) gives a quite thorough statement of this characterization of a thermodynamically reversible process.

Now suppose that a process occurs in which (1) the system proceeds from an initial state i to a final state $\mathrm{f}$; (2) the suspended object is lowered to an extent that W units of work are performed; and (3) a transfer of heat $\mathrm{Q}$ takes place from the system to the series of reservoirs. If, at the conclusion of this process, the system may be restored to its initial state $\mathrm{i}$, the object lifted to its former level, and the reservoirs caused to part with the same amount of heat $\mathrm{Q}$, without producing any changes in any other mechanical device or reservoir in the universe, the original process is said to be reversible. In other words, a reversible process is one that is performed in such a way that, at the conclusion of the process, both the system and the local surroundings may be restored to their initial states, without producing any changes in the rest of the universe. A process that does not fulfill these stringent requirements is said to be irreversible.

Henderson (2014, p. 92) gives a similar definition.

Planck (1897, pp. 82-83, emphasis in original) has a more general version that proceeds indirectly by first defining irreversible processes: 
A process which can in no way be completely reversed is termed irreversible, all other processes reversible. That a process may be irreversible, it is not sufficient that it cannot be directly reversed. This is the case with many mechanical processes which are not irreversible ... The full requirement is, that it be impossible, even with the assistance of all agents in nature, to restore everywhere the exact initial state when the process has once taken place.

\section{The problem}

The difficulty with this characterization is the same as with existential supposition in Section 2.1. One can introduce processes by supposition as a matter of definition. That falls short of showing that they are processes admissible in the theory and that we can infer general results from supposing them, unless they are introduced for purposes of reductio.

The difficulty is sharpened in Zemansky's exposition. Having introduced the above definition, he continued to assert: (p. 192) "We shall show that it is a consequence of the second law of thermodynamics that all natural processes are irreversible." I add the obvious: the laws of thermodynamics also have as consequences that certain perpetual motion machines are impossible. That immediately precludes their supposition, other than for purposes of reductio. Why should thermodynamically reversible processes escape preclusion for the same reason?

\subsection{Processes that are Mechanically Reversible}

As long as we deal only with ordinary thermodynamics, there is little danger that we confuse thermodynamically reversible processes with mechanically reversible processes. These latter are processes whose time reversal is permitted by the governing dynamical laws. The familiar examples are of systems in ordinary mechanics governed by time reversible, conservative Hamiltonians, such as the fully elastic collisions of billiard balls.

Where confusion can arise is when we recall that ordinary thermal systems are composed of many components whose microphysics is mechanically reversible. A gas is not a continuous fluid but many molecules, moving rapidly, undergoing fully elastic collisions, quite like the billiard balls.

That fact invites a conflation of thermodynamic reversibility and mechanical reversibility. The conflation should be avoided. They are quite distinct senses of reversibility. 
- Mechanical reversibility applies to isolated processes that evolve independently of their environment. The mechanical process of elastic billiard ball collisions is reversed merely by momentarily reversing all the velocities of the balls. The process will then proceed to undo itself without further intervention.

- Thermodynamic reversibility applies to processes that evolve in continuous engagement with their environments; and to reverse a thermodynamic process, one must change the entire environment in which the process happens. For example, to reverse an isothermal expansion of a gas in which a weight is raised, one must reconfigure the coupling to the weight so it now overpowers the gas pressure and recompresses the gas. Similar adjustments are needed for the temperatures of the heat reservoirs.

There are other obvious differences. In ordinary mechanical processes, any conversion of work to heat is a dissipative loss. Heat engines, running in reverse, however, can convert work to heat while operating with minimal dissipation in the thermodynamic sense, that is, coming arbitrarily close to a constant entropy process.

We would hope that no one would encourage the conflation of the two senses of reversibility. Unfortunately Planck (1897) does just this. Immediately following his discussion of reversibility as restoration of the initial state, he offers a series of illustrations of mechanical reversibility (p. 83):

... all perfectly periodic processes, e.g. an ideal pendulum or planetary motion, are reversible, for, at the end of every period, the initial state is completely restored. Also, all mechanical processes with absolutely rigid bodies and absolutely incompressible liquids, as far as friction can be avoided, are reversible. By the introduction of suitable machines with absolutely unyielding connecting rods, frictionless joints and bearings, inextensible belts, etc., it is always possible to work the machines in such a way as to bring the system completely into its initial state without leaving any change in the machines, for the machines of themselves do not perform work.

Planck's text proceeds with a further example of a heavy liquid oscillating without friction under gravity in connected tubes.

The invitation to confusion seems largely to have been ignored, although at least one contemporary writer pursued it. Klein (1910, pp. 31-32) quoted Planck's text approvingly and 
then added further examples of his own. They included "Free fall in a vacuum, propagation of light and sound waves without absorption and reflection and unchecked electrical oscillations."

Credit for the most egregrious conflation of the two senses goes to Progogine (1961). His Chapter III "Entropy Production - the Second Principle of Thermodynamics" begins with a first section: (p. 14, emphasis in original)

\section{Reversible and Irreversible Processes}

Let us consider equations which describe some time-dependent physical processes.

If these equations are invariant with regard to the algebraic sign affixed to the variable time, the process is called a reversible process, otherwise it is called irreversible. In the equations describing reversible processes, time can appear only through its arithmetic value.

Lest there be any doubt over the conflation committed, Prigogine then displays a wave equation. It is judged to describe reversible processes, since it is invariant under the substitution of - $t$ for the time variable t. He displays the Fourier equation for temperature changes. It describes an irreversible process since it is not invariant under this substitution. The immediately following section, "2. Entropy," then associates a zero change in entropy with reversible processes and an increase in entropy with irreversible processes.

Prigogine's definition is ruinous to thermodynamics. According to it, the thermodynamically irreversible expansion of heat radiation into empty space is reversible since it is governed by a wave equation. However heat transfer, governed by the Fourier equation, can never be reversible.

There has been a recent literature in philosophy of physics that probes the relation between these senses of reversibility, while, I fear, not always recognizing the how completely distinct they are. See Brown and Uffink (2001), Henderson (2014), Marsland et al. (2014) and Uffink $(2001, \S 11)$.

\subsection{Quasi-static Processes: Possible Meanings}

So far, none of the characterizations of thermodynamically reversible processes can be literally true. Prospects for a new approach came with Carathéodory's (1909) axiomatic approach to thermodynamics. If physicists cannot provide an unobjectionable characterization, perhaps the mathematicians can. Carathéodory (p. 368) remarked that so far the notion of 
"reversible" had been given only intuitively. He would now offer a precise characterization. The core to the new characterization was his notion of "quasi-static process." His concern was the special case of an adiabatic process in a system $\mathrm{S}$ for which the work "A" would be determined by the initial states and time evolution of the system tracked by time-t indexed, changing "deformation coordinates" $\mathrm{x}_{1}(\mathrm{t}), \mathrm{x}_{2}(\mathrm{t}), \ldots, \mathrm{x}_{\mathrm{n}}(\mathrm{t})$ that span the non-equilibrium states of the system. He then posited (p. 366):

... when the velocity with which the system is deformed becomes "infinitely slow," or, more precisely, when the derivatives:

$$
x_{1}{ }^{\prime}(t), x_{2}{ }^{\prime}(t), \ldots, x_{n}^{\prime}(t)
$$

converge uniformly to null, the work A shall go to a definite value in the limit. We would like to call a 'quasi-static' change of state one that proceeds so slowly that the difference between the applied external work and this limiting value falls beneath the limit of observation.

This definition is soon explicated by (emphasis in original):

A quasi-static, adiabatic change of state can thus be interpreted as a sequence of equilibrium points, and each quasi-static, adiabatic change of state corresponds to a specific curve in the space of $x_{i}$.

However careful Carathéodory may have been, his description here proved too terse to fix a definite meaning for quasi-static processes in the subsequent literature. What is striking in that literature is the proliferation of readings for the notion. As the survey below will show, it proves to be a term that should be used with caution. It has no universally agreed upon meaning. We shall even see that many authors distinguish quasi-static processes from reversible processes, which negates Carathéodory's original goal.

There seem to be at least three readings that have been grafted onto Carathéodory's idea. They will be treated separately below. For reference I name them as follows:

1. Bare Curve. A quasi-static process is merely a set of equilibrium states forming a curve in equilibrium state space. 
This reading picks up on Carathéodory's mention of "infinitely slow," of velocities taking their limiting values of zero and the word "curve." 18 A second reading seeks to add a dynamical element, perhaps reflecting Carathéodory's concern with the work term:

2. Force-Balanced Curve. A quasi-static process is a process in which a (near) perfect balance of forces drives the system (near enough) through a continuous set of equilibrium states that form a curve in equilibrium state space.

There are two versions: a strict version without the parenthetic qualifications "near" and "near enough"; and a qualified version with them.

Finally, there is a reading that interprets Carathéodory's "sequence of equilibrium points" as a discrete set connected by curves in a non-equilibrium space of states spanned by the coordinates $\mathrm{x}_{\mathrm{i}}$ :

3. Iterated Equilibria. A quasi-static process is a process in which the system passes very slowly by means of a non-equilibrium process from one equilibrium state to the next, and so on, for a set of equilibrium states, distributed as discrete points along a curve in equilibrium state space.

A helpful image here is of a stone skipping across the water of a pond: it is mostly in the air of non-equilibrium states, touching down only momentarily on the equilibrium state, water surface.

Which of these Carathéodory intended is not entirely clear. Mention of "infinitely slow" and of time derivatives of the coordinates of the non-equilibrium states $x_{1}{ }^{\prime}(t), x_{2}{ }^{\prime}(t), \ldots, x_{n}{ }^{\prime}(t)$ converging to zero suggest equilibria. That indicates the bare or force-balanced curve, implemented strictly. However the persisting of differences from equilibrium conditions "beneath the limit of observation" and a "specific curve in the [nonequilibrium] space of $\mathrm{x}_{\mathrm{i}}$ " suggest the qualified force-balanced curve or the implementation as iterated equilibria.

18 It is all too easy to overlook that Carathéodory's "space of of $\mathrm{x}_{\mathrm{i}}$ " that hosts the curve is not equilibrium state space. Instead, the coordinate $\mathrm{x}_{0}$ is the only equilibrium state coordinate; the remaining coordinates span non-equilibrium states. 


\subsection{Quasi-Static Processes: The Bare Curve}

This bare reading is common. In a review article "Fundamental Thermodynamics Since Carathéodory," Redlich (1968, p. 558) glosses quasi-static processes briefly as "a sequence of equilibrium states." Pauli (1973, p. 29) gives this definition:

3. Quasi-static changes of state: These changes of state are very slow, infinitely

slow in the limiting case, so that the intermediate states form a continuous sequence of equilibrium states.

He makes clear that this notion is to play the role of reversible processes when he remarks (p. 17) "A quasi-static process is always reversible."

In spite of the unambiguity of their words, it is hard to believe that accomplished authors such as these really intend the bare notion to play the role of reversible processes, for the bare notion falls well short of what is needed. We have already seen that mere infinite slowness is not enough to bring reversibility. Moreover, a quasi-static process cannot be represented simply by the resulting parameterized curve in the state space, if it is to serve the function of thermodynamically reversible processes.

What is the problem? An elementary example illustrates it. Consider the isothermal expansion of an ideal gas of $\mathrm{n}$ moles at pressure $\mathrm{P}$, volume $\mathrm{V}$ and temperature $\mathrm{T}$. Its equation of state is $\mathrm{PV}=\mathrm{nRT}$ for the $\mathrm{R}$ the ideal gas constant. If it expands reversibly following the usual prescriptions, then work

$$
\mathrm{dW}=\mathrm{PdV}
$$

is done by the gas pressure $\mathrm{P}$ during a small expansion $\mathrm{dV}$ of the volume. To preclude cooling of the gas from this loss of energy, a quantity of heat dQ must be passed to the gas from the surroundings. Since the internal energy of an ideal gas is fixed by its temperature and that is maintained constant at $\mathrm{T}$, the internal energy will not change and we have $\mathrm{dQ}=\mathrm{dW}$. During this expansion, the pressure will drop according to

$$
\mathrm{P}=\mathrm{nRT} / \mathrm{V}
$$

The process will be traced out by the hyperbola in PV space corresponding to this formula.

The difficulty is that there is a second fully irreversible process that traces out the same curve and can do it as slowly as we like. We once again allow an ideal gas to expand. But this time we employ the closely spaced barriers or membranes mentioned in Section 2.5 above. Or we can employ a cylinder with a piston whose motion is so impeded by friction that it barely 
moves. During the expansion no energy is extracted from the piston as work. All that energy is lost to friction as heat, which is returned to the gas. Since the internal energy remains constant, so does the temperature $\mathrm{T}$ of the ideal gas. This irreversible expansion traces the same hyperbola (12) in the PV state space and, by suitable increase in the friction, can be made to take an arbitrarily long time.

Thus, this characterization of quasi-static processes is inadequate if it is also to represent thermodynamically reversible processes.

The failure of the bare curve is reflected in a continuing literature devoted to correcting the misuse. Samiullah (2007, p. 608) reports

A quasi-static process is a change in the state of a system that is conducted infinitesimally slowly such that, at each instant, the system is in thermodynamic equilibrium with its environment, and its thermodynamic properties, such as volume, pressure, and temperature, remain well-defined throughout the process.

Quasistatic processes are often misunderstood to be reversible, especially in elementary physics texts.

To illustrate a quasi-static process that is not reversible, Thomsen (1960) explores the above example of a gas expanded by a friction-damped piston.

Because of these difficulties, there now is a well-established tradition of distinguishing quasi-static processes, in the bare sense here, from reversible processes. We saw above in Section 7.3 that MacDonald (1995, p. 1122) was able to define "a quasistatic irreversible process" and to require further conditions if a quasi-static process is to be reversible. Lebon et al. (2008, p. 4) characterize a quasi-static process as "those that may be considered as a sequence of neighbouring equilibrium states" and then affirm: "A quasi-static process is either reversible or irreversible." Linder (2004, pp.10-12) briefly surveys reports in the literature that not all quasistatic processes are thermodynamically reversible, concluding that "the terms quasi-static and reversible are not uniquely defined." He gives two definitions of quasi-static process. The second is illustrated by the infinitely slow heating of a system through a poorly conducting metal plate. It is not reversible, I presume, since this heating would have a finite temperature difference across the metal plate. 


\section{The problem}

While a bare curve in equilibrium state space is a well-defined structure, the above discussion demonstrates that it cannot serve the function of a thermodynamically reversible process.

\subsection{Quasi-Static Processes: The Force-Balanced Curve}

The difficulties of the bare curve indicate that the notion of a quasi-static process involves more conditions on the system than can be recovered solely from the curve in equilibrium state space. That is, the curve must be produced in the right way, which is by perfectly balanced or near perfectly balanced forces.

This was a central concern of Carathéodory's original definition. He separated the cases of the hyperbola (12) representing a reversible or an irreversible expansion of the gas by requiring that a quantity of work corresponding to (11) be associated with the process. For this quantity of work is returned by the reversible process, but not the irreversible process. This work term, in German Arbeit, A(t), can be expressed solely as a function of the state variables defined along the curve, which is parameterized by a variable $t$ :

$$
A(t)=\int_{t_{0}}^{t} D A
$$

where the Pfaffian DA is the generalization for many variables of the work term dW of (11)

$$
\mathrm{DA}=\mathrm{p}_{1} \mathrm{dx}_{1}+\mathrm{p}_{2} \mathrm{dx}_{2}+\ldots+\mathrm{p}_{\mathrm{n}} \mathrm{dx}_{\mathrm{n}}
$$

This strict, force-balanced formulation is quite common. Wilson $(1957$, p. 10) offers it as a formal definition:

A quasi-static change in which a body passes from the equilibrium state 1 to the equilibrium state 2 is such that there is a linear continuum of equilibrium states to which 1 and 2 belong, and such that the body will successively take the states connecting 2 to 1 by a simple reversal of sign of the displacements, of the work done and the heat absorbed.

The balance of forces is reflected in the requirement that the process reverses with the given sign reversals. This version is the strict one without the parenthetic "near enough" qualification. As a result, Wilson must allow (p. 9) that such a process "cannot be realized in practice, though we may approximate to it in favorable circumstances." Comparable versions can be found in 
Buchdahl (1966, p. 11), Linder (2004, p. 11), Pippard (166, p. 21) and in Reiss (1965, p. 9), who takes this sense of quasi-static to be synonymous with reversible.

The qualified force-balanced formulation is rarer. Here is a version in Zemansky (1968, p. 53; his emphasis):

We are led, therefore, to conceive of an ideal situation in which the external forces acting on a system are varied only slightly so that the unbalanced force is infinitesimal. A process performed in this ideal way is said to be quasi-static.

During a quasi-static process, the system is at all times infinitesimally near a state of thermodynamic equilibrium, and all states through which the system passes can be described by means of thermodynamic coordinates referring to the system as a whole.

Zemansky continues to note that such process cannot be realized in the laboratory but can be approximated arbitrarily well.

\section{The problem}

The difficulty for the strict version is that the process has become a purely mathematical construction. It is not a real physical process in which a system changes its state through time, no matter how earnestly we name it so. Callen (1985, p. 96, emphasis in original) makes the point with clarity:

A quasi-static process is thus defined in terms of a dense succession of equilibrium states. It is to be stressed that a quasi-static process therefore is an idealized concept, quite distinct from a real physical process, for a real process always involves nonequilibrium intermediate states having no representation in the thermodynamic configuration space. Furthermore, a quasi-static process, in contrast to a real process, does not involve considerations of rates, velocities, or time. The quasi-static process simply is an ordered succession of equilibrium states, whereas a real process is a temporal succession of equilibrium and nonequilibrium states.

There is a similar problem, I add, with the assignment of the work term (13) in the strict version. It is a purely abstract attachment. There is no transfer of work energy. For a force only does work when the force moves through a distance and there is no such motion. One can integrate 
Carathéodory's generalized forces $\mathrm{p}_{\mathrm{n}}$ of (14) in (13). But since nothing is moving or changing state in time, there is no work associated with it. There is just an integrand.

The qualified version escapes these difficulties. It allows imbalances of forces that can drive a real process. However it faces the familiar difficulty developed in Sections 7.2, 7.3 and 7.4 that the states are not equilibrium states, even if "infinitesimally" close to them. This concern is presumably the import of Truesdell's $(1969$, p. 22) riposte "... whatever it is that the thermodynamicists mean by "quasi-static"". To it he adds, in an ironic footnote, the unsourced quote " 'A change so small that the system may be considered in equilibrium at all times' (sic)."

\subsection{Quasi-static processes: Iterated Equilibria}

A sustained attempt to clarify Carathéodory's notion of quasi-static process was made by Tatiana Ehrenfest-Afanassjewa (1956). ${ }^{19}$ In her earlier analysis (1925, p. 934), she had used a bare characterization: "We call--following Caratheodory--a continuous sequence of pure equilibrium states a "quasi-static" change of state." This reflects the difficulties even careful writers have had in interpreting Carathéodory's work. By the time of her later work, this bare characterization was replaced by a carefully made distinction between quasi-processes and quasistatic processes.

A "quasi-process" coincides with the bare curve: it is just a continuous set of equilibrium states forming a curve in the equilibrium state space. She writes (1956, p. 13):

We will very much concern ourselves further with continuous sequences of equilibrium states that connect two given equilibrium states. They will be represented graphically by curves in $R_{n}$ [equilibrium state space]. They have so far always had the name of "processes" and indeed of "reversible" processes. We will prefer to call them "quasi-processes," for-obviouslyone can realize them through no real process; however in this regard, we want to discard emphatically the epithet "reversible"(see $§ 10) .{ }^{20}$

19 I thank Jos Uffink for drawing this important, less known work to my attention.

20 In $§ 10$, she explains further: “...quasi-processes just aren’t processes. There is, therefore, nothing there that could be reversed..." 
Since a quasi-process is no process at all, Ehrenfest-Afanassjewa introduced the distinct notion of a quasi-static process to connect quasi-processes with real processes. Their importance is most fully explained later in the text (p. 56):

In order to make a quasi-process open to experimental investigation or just to connect it with experiments in thought, one has to conceive it as approximated by quasi-static processes. These are in turn real processes, if also idealized.

What are quasi-static processes? Some preparatory work had already been done in the section preceding the introduction of this second notion of process. There she (pp. 12-13) defined an "elementary quasi-static process." This process is a "forced change of state" that results from the disturbance from equilibrium in some system by a coupling with the environment. Its properties included the following:

the differences between the initial and final parameters of the given system are arbitrarily small; the state of the system during the entire process is as good as an equilibrium state...

as well as further conditions that limit the speed with which the parameters characterizing the system can change.

A quasi-static process is then introduced as a compounding of a sequence of elementary quasi-static processes (p. 13):

Although a quasi-process, strictly speaking, is therefore not realizable, one can at least posit an arbitrarily dense, discrete sequence of equilibrium states that belong to the system, and indeed such, that also during the process that connects two consecutive equilibrium states, the state of the system is well-nigh an equilibrium state. That is to say, one allows the system to pass through a sequence of elementary quasi-static processes. We call such a sequence a "quasi-static process." (compare C. Caratheodory, Untersuchung über die Grundlagen der Thermodynamik (Math. Ann. 67[1909], 355)).

In brief, in a quasi-static process, a system passes through a dense, discrete set of equilibrium states with processes whose states are well-nigh (German: beinah) equilibrium states. It is the iterated equilibria conception.

Ehrenfest-Afanassjewa proceeds to note that these processes are "infinitely slow." She had earlier introduced the notion of an infinitely slow process with necessary caution: (p.11) 
Of course an exact, infinitely slow process does not exist. Hence one cannot say that the just described arbitrarily slow process has an infinitely slow process as a limiting case. ... If we still apply the name "infinitely slow" to the just described process, then we mean to say thereby that the process at issue is sufficiently slow that we may neglect thereby certain deviations in each of its states from equilibrium states.

This conception can be found elsewhere in the literature. Callen (1985, pp. 96-97) gives a careful exposition of the process of iterated equilibria, complete with a diagram in which the equilibrium states of the process are represented as points on a surface in equilibrium state space. Callen, however, does not call the process of iterated equilibria a quasi-static process. That term is reserved for the curve drawn through the points in equilibrium state space. His sense corresponds with the force-balanced curve. Landsberg (1978, p. 5) has the same formulation. Having introduced thermodynamic state variables, he continues:

By changing the variables and after each change allowing the system to return to equilibrium before one is estimating the values of the variables, one can plot various states characteristic of the systems in the thermodynamic phase spaces.

Curves drawn through these points are such that each point represents an equilibrium state. One can imagine a system being taken slowly through all the states represented on such a curve. This process is called a quasi-static process. O'Connell and Haile (2005, p. 21) describe the same processes and structures, but label them differently. They define a quasi-static process as one with non-equilibrium jumps between equilibrium states. They do not, however, identify their quasi-static processes with reversible processes. The latter are produced by taking a limit in which all imbalanced driving forces are set to zero. "Hence," they conclude, "a reversible change can be represented by a continuous line on a state diagram."

\section{The problem}

Quasi-static processes as iterated equilibria are real processes unfolding in time. They depend on just the troublesome notions we have seen in other treatments. The states through which the process passes are not equilibrium states, but they are so close that they are "as good as" or "well-nigh." The processes are said to proceed infinitely slowly. Fortunately Ehrenfest- 
Afanassjewa allows that there is no cogent notion of infinitely slow process as a limit. Rather they are processes that are so slow that we may neglect deviations of equilibrium.

There is a further problem arising from the requirement that the processes jump successively from equilibrium state to nearby equilibrium state. In generic systems, no single jump can complete, if it is to terminate in an equilibrium state. Informally speaking, as the process approaches its equilibrium end state, the driving forces drop to zero. The result, at least in the generic case, is that the approach is asymptotic, coming arbitrarily close to completion, but never arriving there in any finite time.

For example, consider a body with heat capacity $\mathrm{C}$ at temperature $\mathrm{T}$ cooling to equilibrium at temperature $\mathrm{T}_{\mathrm{e}}$ by losing heat to a heat bath at $\mathrm{T}_{\mathrm{e}}$. The rate of heat transfer is $k\left(T-T_{e}\right)$, for heat transfer coefficient k. Combining, the process is governed by the differential equation

$$
\frac{d}{d t} C\left(T-T_{e}\right)=-k\left(T-T_{e}\right)
$$

The solution is an asymptotic approach to the equilibrium temperature $\mathrm{T}_{\mathrm{e}}$ :

$$
T(t)=T_{e}+\left(T(0)-T_{e}\right) \exp \left(-\frac{k}{C} t\right)
$$

where $\mathrm{T}(0)$ is the body's temperature at $\mathrm{t}=0$.

For moving bodies, such as pistons, a comparably simple case is a body brought to rest by velocity dependent friction forces. Generically, such a motion is governed in its final stages close to equilibrium by the linearized differential equation $\frac{d}{d t} m v=-\mu v$, where the body has mass $\mathrm{m}$, speed $\mathrm{v}$ and coefficient of friction $\mu$. The solution is an exponential decay of the same form that allows only an asymptotic approach to rest at $\mathrm{v}=0$.

$$
v(t)=v(0) \exp \left(-\frac{\mu}{m} t\right)
$$

\section{Conclusion}

The principal claim of this paper is that the difficulties of thermodynamically reversible processes lie purely in the definitions used. There is no irreparable failure of thermodynamic theory. There is a failure only of our descriptions of one of its central notions. Section 7 above 
gives an inventory of the problems of standard definitions, most of which derive from the tensions between the two parts of the paradox outlined in Section 1.

In so far as a thermodynamically reversible process is constituted of equilibrium states, it is no real process of change, even as an invention in imagination of a highly idealized fiction. For such a process would contradict basic facts of ordinary mechanics and of thermal physics. It would transfer heat without a temperature difference and move bodies mechanically without a net, non-zero force.

In so far as thermodynamically reversible process is constituted of non-equilibrium states, it cannot have the properties of equilibrium systems. There is no remedy in stipulations that the states of the process are so close to equilibrium, even infinitesimally close, that the difference does not matter. For the difference does matter: it is what enables the system to change in time. Otherwise the difference could be eliminated.

The proposal of Section 2 is designed specifically to resolve the paradox and to avoid the problems listed in Section 7 above. Key elements in the proposal are:

- The term "thermodynamically reversible process" does not designate a process constituted by equilibrium states, but a set of irreversible processes, all constituted of non-equilibrium states.

This eliminates the difficulty that equilibrium states do not change with time and thus cannot partake in a process of change.

- The irreversible processes form a set whose states come arbitrarily close to equilibrium states.

Characterizing a reversible process as a single process leads to the difficulty of determining just how close the states of the single process should come to equilibrium to be close enough for the driving forces to vanish near enough and for quantities of heat and work to adopt reversible values. Employing a set rather than a single process eliminates the difficulty: these properties are recovered as limit properties of the set, without the need for qualifications like "near enough." The limit operation generates properties only. It does not generate a single process with the internally contradictory properties of being at equilibrium yet still changing.

- The notion of reversibility is implemented by the existence of both "forward" and "reverse" processes in the set. 
Otherwise reversibility is represented inadequately in the fact that a continuous set of unchanging equilibrium states can be reparameterized in the reversed direction; or in the vague locution that a single irreversible process that is "near enough" to equilibrium states can be reversed by suitably small change in driving forces.

Finally, thermodynamically reversible processes have always functioned to demarcate an

unrealizable boundary of our real interest: the least dissipative processes of change. The proposal of Section 2 employs these least dissipative processes directly. Using them, Section 4 shows how we can recover standard results concerning the boundaries of these processes by reasoning directly with the irreversible processes of the set, without any need to talk of processes that, paradoxically, are both in and not in equilibrium at the same time.

\section{References}

Atkins, Peter (2010) The Laws of Thermodynamics: A Very Short Introduction. Oxford: Oxford University Press.

Brown, Harvey R. and Uffink, Jos (2001) “The Origins of Time-Asymmetry in Thermodynamics: The Minus First Law," Studies in History and Philosophy of Modern Physics. 32, pp. 525-38.

Buchdahl, Hans A. (1966) The Concepts of Classical Thermodynamics. Cambridge: Cambridge University Press.

Callen, Herbert B. (1985) Thermodynamics and an Introduction to Thermostatistics. 2nd ed. New York: John Wiley \& Sons.

Carathéodory, Constantin (1909) "Untersuchungen über die Grundlagen der Thermodynamik," Mathematische Annalen 67, pp. 355-386. Translated by D. H. Delphenich as "Examination of the Foundations of Thermodynamics," http://neo-classicalphysics.info/uploads/3/0/6/5/3065888/caratheodory_-_thermodynamics.pdf Carnot, Lazare (1786) Essai sur les Machines en Général. Dijon: De Defay. Carnot, Lazare (1808) "Essay upon Machines in General," Philosophical Magazine. 30, pp. 8-15, 154-58, 207-221, 310-320; 31, pp. 28-36, 136-146, 220-228, 295-305. 
Carnot, Sadi (1824) Réflexions sur la puissance motrice du feu. Paris: Bachelier. Trans in Reflections on the Motive Power of Heat. New York: John Wiley \& Sons, 1897. Clapeyron, Émile (1837) "Memoir on the Motive Power of Heat," From the Journal de l'Ecole Royale Polytechnique; Paris; vol. xiv.p. 153 et seq. pp. 347- 376 in Scientific Memoirs. R. Taylor, ed. Vol. III. London: Richard and John E. Taylor.

Clausius, Rudolf (1851) "On the Moving Force of Heat, and the Laws Regarding the Nature Heat Itself which are Deducible Therefrom,” Philosophical Magazine, 2 (ser. 4), pp. 1$21,102-119$.

Clausius, Rudolf (1865) "Ueber verschiedene für die Anwendung bequeme Formen der Hauptgleichungen des mechanischen Wärmetheorie," Annalen der Physik und Chemie, 125, pp. 353-400.

Clausius, Rudolf (1879), The Mechanical Theory of Heat. Trans. W. R. Browne. London: MacMillan \& Co.

Duhem, Pierre (1898) "On the General Problem of Chemical Statics," Journal of Physical Chemistry, 2, pp. 1-42, 91-115.

Duhem, Pierre (1903) Thermodynamics and Chemistry: A Non-Mathematical Treatise for Chemists and Students of Chemistry. Trans. G. K. Burgess. New York: John Wiley \& Sons.

Ehrenfest-Afanassjewa, Tatiana (1925) "Zur Axiomatisierung des zweiten Hauptsatzes der Thermodynamik," Zeitschrift für Physik, 33, pp. 933-45; 34, p. 638.

Ehrenfest-Afanassjewa, Tatiana (1956) Die Grundlagen der Thermodynamik. Leiden: E. J. Brill. Fermi, Enrico (1937) Thermodynamics. New York: Prentice-Hall. Reprinted New York: Dover, 1956.

Goodenough, George A. (1911) Principles of Thermodynamics. New York: Henry Holt and Co. Henderson, Leah (2014) "Can the second law be compatible with time reversal invariant dynamics?" Studies in History and Philosophy of Modern Physics, 47, pp. 90-98.

Kestin, Joseph (1979) A Course in Thermodynamics. Vol 1. Levittown, PA: Hemisphere. Klein, Joseph F. (1910) Physical Significance of Entropy. New York: D. Van Nostrand Co. Landau, L. D. and Lifshitz, E. M. (1970) Statistical Physics. 2nd ed. Trans. J. B. Sykes and M. J. Kearsley. Oxford: Pergamon Press. 
Landsberg, Peter T. (1978) Thermodynamics and Statistical Mechanics. Oxford: Oxford University Press; New York: Dover, 1990.

Lebon, G., Jou, D. and Casas-Vázquez (2008) Understanding Non-equilibrium Thermodynamics. Berlin: Springer.

Lewis, Gilbert N. and Randall Merle (1923) Thermodynamics and the Free Energy of Chemical Substances. New York: McGraw-Hill.

Lieb, Elliott and Yngvason, Jakob (1998) “A Guide to Entropy and the Second Law of Thermodynamics," Notices of the American Mathematical Society, 45, pp. 571-81.

Lieb, Elliott and Yngvason, Jakob (1999) "The Physics and Mathematics of the Second Law of Thermodynamics," Physics Reports 310, pp. 1-96.

Linder, Bruno (2004) Thermodynamics and Introductory Statistical Mechanics. Hoboken, NJ: Wiley-Interscience.

Macaulay, William. H. (1913) The Laws of Thermodynamics. Cambridge: Cambridge University Press.

Marsland III, Robert, Brown, Harvey and Valente, Giovanni (2014) “Ambiguities in ordertheoretic formulations of thermodynamics." http://philsci-archive.pitt.edu/10916/

Maxwell, James C. (1872) Theory of Heat. New York: D. Appleton \& Co.

MacDonald, Alan (1995) “A New Statement of the Second Law of Thermodynamics," American Journal of Physics, 63, pp. 1122-27.

Needham, Paul (ed.) (2011) Commentary on the Principles of Thermodynamics by Pierre Duhem. Boston Studies in the Philosophy of Science. Vol 277. Springer.

Needham, Paul (2013) “Process and Change: From a Thermodynamic Perspective," British Journal for the Philosophy of Science, 64, pp. 395-422.

Norton, John D. (1998) How Science Works. McGraw-Hill Higher Education.

Norton, John D. (2004) "Why Thought Experiments Do Not Transcend Empiricism" pp. 44-66 in Christopher Hitchcock (ed.) Contemporary Debates in the Philosophy of Science. Blackwell.

Norton, John D. (2012) "Approximation and Idealization: Why the Difference Matters" Philosophy of Science, 79, pp. 207-232.

Norton, John D. (2013) "All Shook Up: Fluctuations, Maxwell's Demon and the Thermodynamics of Computation," Entropy, 15, pp. 4432-4483. 
Norton, John D. (2013a) "The End of the Thermodynamics of Computation: A No-Go Result," Philosophy of Science, 80, pp. 1182-1192.

Norton John D. (2014) "Infinite Idealizations," European Philosophy of Science--Philosophy of Science in Europe and the Viennese Heritage: Vienna Circle Institute Yearbook, Vol. 17 (Springer: Dordrecht-Heidelberg-London-New York), pp. 197-210.

Norton, John D. (forthcoming) "Thermodynamically Reversible Processes in Statistical Physics." American Journal of Physics.

O'Connell, John P. and Haile, J. M. (2005) Thermodynamics: Fundamentals for Applications. Cambridge: Cambridge University Press.

Pauli, Wolfgang (1973) Pauli Lectures in Physics: Volume 3. Thermodynamics and the Kinetic Theory of Gases. Cambridge, MA: MIT Press.

Pippard, Alfred B. (1966) Elements of Classical Thermodynamics for Advanced Students of Physics. Cambridge: Cambridge University Press.

Porter, Alfred W. (1931) Thermodynamics. London: Methuen.

Poynting, John H. and Thomson, Joseph John (1920) A Text-Book of Physics: Heat. 6th ed. London: Charles Griffith \& Co.

Prigogine, Ilya (1961) Introduction to Thermodynamics of Irreversible Processes. 2nd ed. Interscience Publishers, New York: John Wiley \& Sons.

Rechel, Ernest R. (1947) “The Reversible Process in Thermodynamics,” Journal of Chemical Education, 24, pp. 298-301.

Redlich, Otto (1968) "Fundamental Thermodynamics since Carathéodory," Reviews of Modern Physics 40, pp. 556-563.

Reiss, Howard (1965) Methods of Thermodynamics. New York: Blasidell Publishing.

Röntgen, Robert (1880) The Principles of Thermodynamics with Special Applications to Hot-Air, Gas and Steam Engines. Translated, revised and enlarged, A. Jay du Bois. New York; John Wiley \& Sons.

Samiullah, Mohammad (2007) “What is a reversible process?” American Journal of Physics, 75, pp. 608-609.

Sommerfeld, Arnold (1956) Thermodynamics and Statistical Mechanics. Trans. J. Kestin. New York: Academic Press. 
Sommerfeld, Arnold (1962) Thermodynamik und Statistik. 2nd edn. Harri Deutsch, Frankfurt, 2002.

Tait, Peter G. (1877) Sketch of Thermodynamics. 2nd ed. Edinburgh: David Douglas.

Trevor, Joseph E. (1927) The General Theory of Thermodynamics. Boston: Ginn and Company.

Thomsen, John S. 1960) "Thermodynamics of an Irreversible Quasi-Static Process," American Journal of Physics, 28, pp.

Thomson, William (1848) "On an Absolute Thermometric Scale," Cambridge Philosophical Society, Proceedings, June 5, 1848; reprinted as pp. 100-106 in William Thomson, Mathematical and Physical Papers, Vol. 1. Cambridge: Cambridge University Press, 1882.

Thomson, William (1849) "Carnot's Theory of the Motive Power of Heat." Transactions of the Edinburgh Royal Society, xiv. Reprinted as commentary in Sadi Carnot, Reflections on the Motive Power of Heat. New York: John Wiley \& Sons, 1897, Ch. IV.

Thomson, William (1852) "On the Dynamical Theory of Heat, with Numerical Results Deduced From Mr. Joule's Equivalent of a Thermal Unit, and M. Regnault's Observations on Steam," 4 (Ser. 4), pp. 8-21, 105-117, 168-176, 256-260.

Truesdell, C. (1969) Rational Thermodynamics. New York: McGraw-Hill.

Uffink, Jos (2001) "Bluff Your Way in the Second Law of Thermodynamics," Studies in History and Philosophy of Modern Physics, 32, pp. 305-394.

Uffink, Jos (2006) "Three Concepts of Irreversibility and Three Versions of the Second Law" pp. 275-287 in F. Stadler, M. Stöltzner (eds.), Time and History. Zeit und Geschichte. Frankfurt: Ontos Verlag.

Van Ness, Hendrick C. (1969) Understanding Thermodynamics. New York: McGraw-Hill. Reprinted, New York: Dover, 1983.

Wilson, A. H. (1957) Thermodynamics and Statistical Mechanics. Cambridge: Cambridge University Press.

Zemansky, Mark W. (1966) “Kelvin and Carathéodory-A Reconciliation,” American Journal of Physics, 34, I pp. 914-920.

Zemansky, Mark W. (1968) Heat and Thermodynamics An Intermediate Textbook. 5th ed. New York: McGraw-Hill. 